\title{
Machine learning prediction Models of Electrical Efficiency of Photovoltaic-Thermal Collectors
}

\author{
Mohammad Hossein Ahmadi', Alireza Baghban', Ely Salwana ${ }^{3}$, Milad Sadeghzadeh $^{4}$, \\ Mohammad Zamen ${ }^{5}$, Shahaboddin Shamshirband6,7, Ravinder Kumar ${ }^{8}$
}

\begin{abstract}
1,5 Faculty of Mechanical Engineering, Shahrood University of Technology, Shahrood, Iran
2 Chemical engineering Department, Amirkabir University of Technology, Mahshahr Campus, Mahshahr, Iran

3 Institute of Visual Informatics, Universiti Kebangsaan Malaysia 43600 Bangi,

Selangor, Malaysia.
\end{abstract}

4 Department of Renewable Energies, Faculty of New Sciences and Technologies, University of Tehran, Tehran, Iran

6 Department for Management of Science and Technology Development, Ton Duc Thang University, Ho Chi Minh City, Vietnam

7 Faculty of Information Technology, Ton Duc Thang University, Ho Chi Minh City, Vietnam

${ }^{8}$ Department of Mechanical Engineering, Lovely Professional University, Punjab, India-14411

Email address of the corresponding author:

shahaboddin.shamshirband@,tdtu.edu.vn (S.Shamshirband)

\begin{abstract}
Solar energy is a renewable resources of energy which is broadly utilized and have the least pollution impact between the available alternatives of fossil fuels. In this investigation, machine leaening approaches of neural networks $(\mathrm{NN})$, neuro-fuzzy and least squares support vector machine (LSSVM) are used to build the models for prediction of the thermal performance of a photovoltaic-thermal solar collector (PV/T) by estimating its efficiency as an output of the model while inlet temperature, flow rate, heat, solar radiation, and heat of sun are input of the designed model. Experimental measurements was prepared by designing a solar collector system and 100 data extracted. Different analyses are also performed to examine the credibility of the introduced approaches revealing great performance. The suggested LSSVM model represented the
\end{abstract}


best performance regarding the mean squared error (MSE) of 0.003 and correlation coefficient $\left(\mathrm{R}^{2}\right)$ value of 0.99 , respectively.

Keyword: PV/T collector; Electrical efficiency; Renewable energy; intelligent models; optimization; machine learning; multilayer perceptron (MLP), artificial neural network (ANN); adaptive neuro-fuzzy inference system (ANFIS); least squares support vector machine (LSSVM); photovoltaic-thermal (PV/T)

\section{Introduction}

Developing more efficient systems and utilizing other energy resources are taking more significance since the amount of accessible fossil fuel resources are facing a decreasing slope. There are several renewable energy sources which can be exploited to satisfy the energy sector demands. However, solar energy is considering more attention since it is available almost everywhere and also it is considered as clean energy with no harmful effect on the environment [1-4]. There are two defined classifications of active and passive for utilizing solar energy. in the passive approach, there is no requirement to any extra equipment and sun irradiations are directly utilized. While in the latter, existence of mechanical components are necessary for solar energy utilization and the conversion process of solar energy to another form of energy is not direct. Solar collectors are classified in the active approach of solar energy conversion to a targeted type of energy $[2,5-8]$.

A solar collector is defined as an equipment which is used to gather sun-rays and absorb sunlight thermal energy and delivered it to a working fluid, mostly air or water. The transferred thermal energy in the working fluid can be stored in a storage tank to be used when solar energy is not sufficient or is not available (e.g. during the nights). Photovoltaic panels use solar irradiations and produce electricity. Moreover, during this electricity production process, considerable amount of 
waste heat is also generated which can be taken its benefit by integrating a network of tubes which containing a fluid for heat transfer process $[9,10]$.

Solar collectors can be categorized into two classifications based on the tracking model: no tracking system is installed, fixed collectors, and a tracker system is provided for tracking the sunlight during the daylight, tracking collectors. There is no movement for the fixed collectors, while the tracking collectors move in a way where the incoming sun-rays are perpendicular to the surface of the collectors. Flat plate collectors, evacuated tube collector are classified as the fixed collector. There are two subclasses of single axis tracking and double axis tracking for the tracking solar collectors. The former is classified into three groups of parabolic and cylindrical trough collectors and linear Fresnel collectors. The latter examples are central tower collectors, parabolic dish collectors, and circular Fresnel lenses. All of the mentioned technologies have their specific applications based on the feasibility of the required and available amount of energy demand and also some other climatic considerations [11-13].

Currently, several methods are developed to predict the quantity of the solar energy production. The major methods are classified in the two approaches of the cloud imagery integrated with physical models and the machine learning approaches. The prediction horizon is the distinction making factor for selection between the methods. However, there is no unity for the all methods predictions and the accuracy and precisions are different. Different methods are developed for solar irradiance prediction based on the favorite prediction time. [14-18].

Recently, advantages of several PV/T collector systems have been highlighted in the investigations [19]. The market development of various solar thermal collectors were studied and compared with PV solar farms [20]. To avoid time-consuming and also expensive experimental examinations in 
the PV/T systems, soft machine-based forecasting methods are developed. These models are able to forecast the output efficiently based on some required input data. The data are then trained based on the algorithms to predict the desired output. Utilizing the artificial intelligence is increasingly become popular in the fields of heat transfer, e.g. the thermal performance of solar air collectors have been predicted through an ANN approach where the model reported promissing [21]. Varol et al.[22] modified the prediction technique and evaluated three soft computing techniques of ANN, Support Vector Machines (SVM) and ANFIS to forecast the thermal performance of the solar air collectors.

Now, modern computational techniques are developed for optimization purposes, finding the governing functions or solution of actual engineering problems in different disciplines [23-25].

In this research, soft-computational techniques were employed to forecast the thermal performance of the photovoltaic thermal (PV/T) collector. These selected approaches are namely, multilayer perceptron artificial neural network (MLP-ANN), adaptive neuro-fuzzy inference system (ANFIS), and least squares support vector machine (LSSVM). Inlet temperature, flow rate, and available solar thermal flux were considered as input and were trained in the designed smart models to yield the efficiency of the collector as the output.

\section{Theory}

\subsection{ANFIS model}

The momentum duty of the adoptive neuro-fuzzy inference (ANFIS) is to discover for fuzzy decision guidelines in the feed-forward framework. The establishment of conventional ANFIS on the basis of $1^{\text {st }}$ order Takagi-Sugeno inference model is demonstrated in the following figure, Fig. 1. 


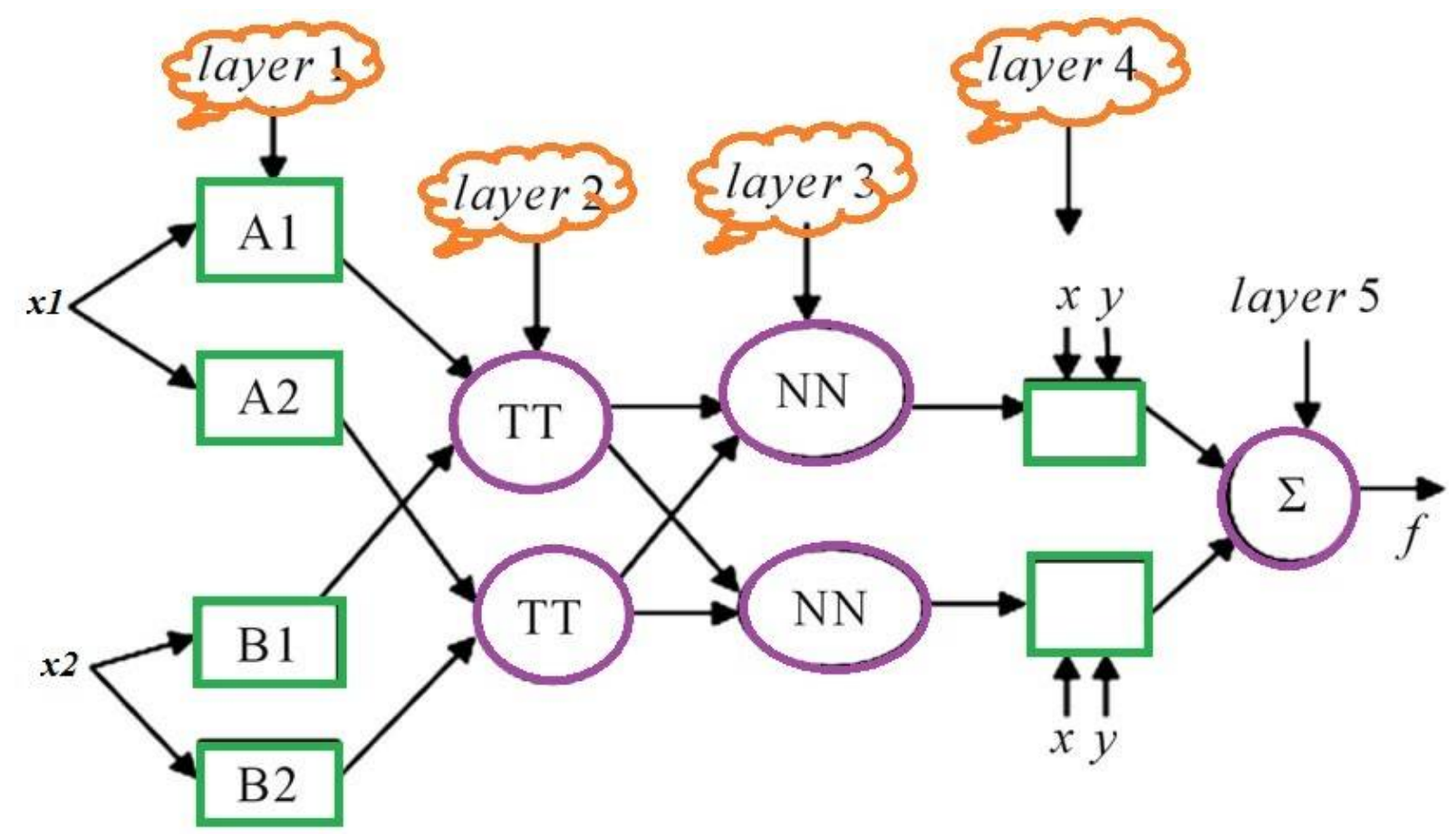

Figure 1: Establishment of typical ANFIS

The ANFIS model states that a primary regulation is made of 5 layers. As shown on figure 1 , inputs of $\mathrm{x}$ and $\mathrm{y}$ are fed into the built model and the following output of $f$ is resulted. In this mode, two different if-then fuzzy statement are defined as follows [26,27]:

Rule 1: If $x$ is $\alpha 1$ and $y$ is $\beta 1$; then $f 1=m 1 x+n 1 y+r 1$

Rule 2: If $x$ is $\alpha 2$ and $y$ is $\beta 2$; then $f 2=m 2 x+n 2 y+r 2$

where $\alpha_{1}, \alpha_{2}, \beta_{1}$, and $\beta_{2}$ are the fuzzy sets for $\mathrm{x}$ and $\mathrm{y}$. Furthermore the varibles of $m_{1}, n_{1}$, $r_{1}, m_{2}, n_{2}$, and $r_{2}$ represent the final outputs of training workflow.

The node functions are defined in every layer as follows:

Layer $I$ is the fuzzfication of the task. Each node $i$ represents an node which is adaptive. The outcome of each node in this layer is: 


$$
O_{1, i}=\mu_{\alpha i}(x) \quad \text { for } i=1,2
$$

$O_{1, i}=\mu_{\beta(i-2)}(y) \quad$ for $i=3,4$

$x$ and $y$ are the node's input data, $i . \mu_{\alpha i}$ and $\mu_{\beta i}$ are functions for the fuzzy membership.

Layer II: devoted to managing the layer and nodes with constant $(i=M)$. The receiving signals are consequently producted and resulted the output. The output is calculated by applying the following equation:

$O_{2, i}=W_{i}=\mu_{\alpha i}(x) \mu_{\beta i}(y) \quad$ for $i=1,2$

Layer III is defined as the normalization layer. The normalized data of the $i^{\text {th }}$ node, N, calculate the normalized strength as follows:

$O_{3, i}=\bar{w}_{i}=\frac{W i}{W 1+W 2} \quad$ for $i=1,2$

Layer IV is configured to de-fuzzy the data. Where between every node, $i$, and a node function an adaptive relation is defined:

$O_{4, i}=\bar{w}_{i} f_{i}=\bar{w}_{i}\left(m_{i} x+n_{i} y+r_{i}\right)$

The parameter sets of this node are $m_{i}, n_{i}$ and $r_{i}$, respectively.

Layer $\boldsymbol{V}$ is the final layer. The overall output of all receiving signals are calculated by a fixed node of $E$ in this layer and then are summed:

$O_{5, i}=\sum_{i} \bar{w}_{i} f_{i}=\frac{\sum_{i} w_{i} f_{i}}{\sum_{i} w_{i}}$

\subsection{MLP}


Artificial neural network (ANN) approach composed of three layer of input layer, output layer, and hidden layer [28-30]. Each layer include some nodes which are called as neurons. Every neurons are connected through an interconnection relationships. A weighted parallel connecting establishment is made to treat these relationships. Multi-layer perceptron, self-organizing map, multilayer recurrent, and cellular neural networks are the typically employed approaches. Among aforementioned layouts, the multi-layer ANN are more distinguished. A conventional layout of the multi-layer ANN is demonstrated in Fig. 2.

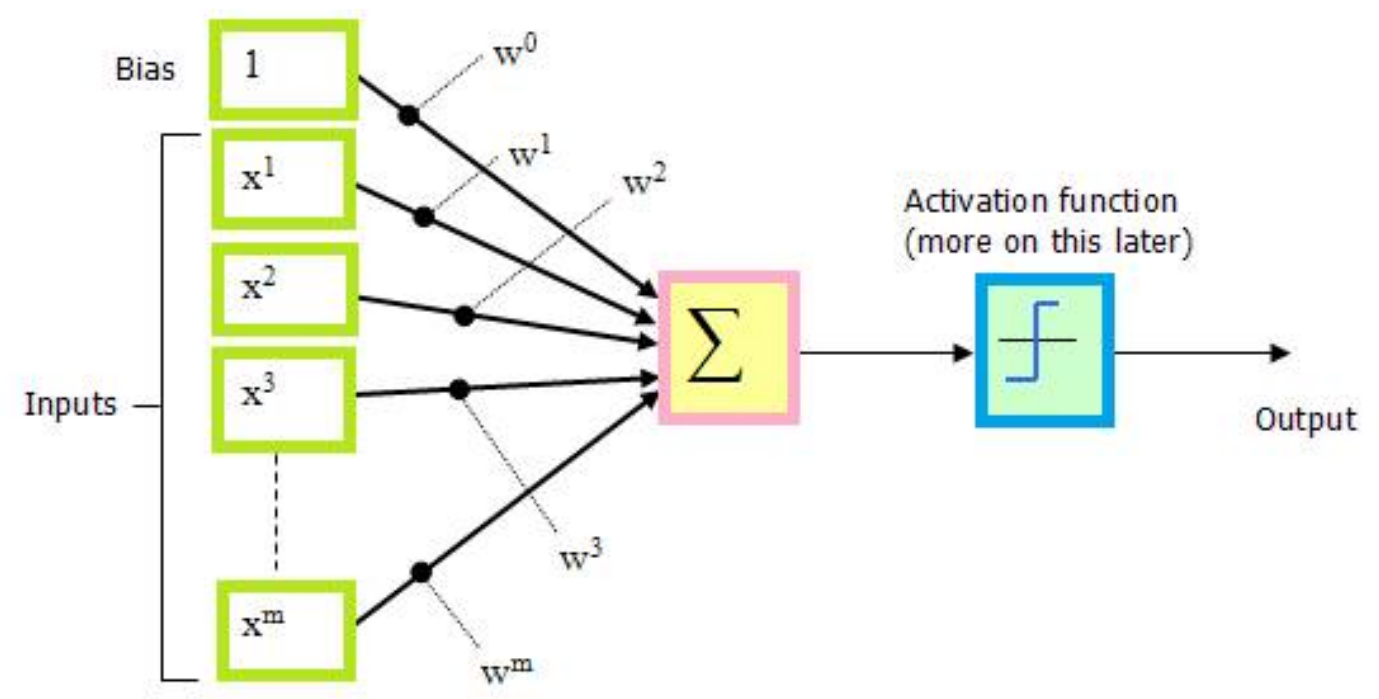

Figure 2: Construction of MLP-ANN model

The two significant parameters in the ANN problems are the weight and the bias. Weight values perform the interconnections throughout the neurons. Moreover, the bias parameter is used to specify the system's degree of freedom (DOF). In the ANN arrangement, the output of every single layer are summed with the values of biases. Then, in order to convert and sent the obtained 
signals to the following layer, transfer function must be used. Linear, Sigmoid, and Hyperbolic tangent functions are known as the most typical transfer function in ANN structures:

- Linear function:

$$
f(z)=z
$$

- Sigmoid function:

$$
f(z)=\frac{1}{1+e^{-z}}
$$

- Hyperbolic tangent function:

$$
f(z)=\frac{e^{z}-e^{-z}}{e^{z}+e^{-z}}
$$

In this investigation, the Sigmoid transfer function, Eq. (10), is employed in the hidden layer and the Linear transfer function, Eq. (9), is applied in the output layer. Thus, the model outcome is obtained as $[31,32]$ :

$$
Z=\sum_{i=1}^{n} w_{3 i} \frac{1}{1+e^{-\left(x_{i} w_{i}\right)}}+b_{3}
$$

Where, $\mathrm{w}_{\mathrm{i}}$ denotes the weight values, $\mathrm{n}$ represents the quantity of neurons in the hidden layer, $\mathrm{w}_{\mathrm{i}, 3}$ indicates the weight values and $\mathrm{b}_{3}$ is the bias. The final outcome is named as $\mathrm{Z}$.

Moreover, the layout of the ANN is trained and is gone through an optimization process by utilizing the Back Propagation (BP) algorithm. During the training stage, the optimum statuses of weights and biases are calculated. While biases and weights reach to their optimum values, the disparity of the prediction of the ANN model and the real measured data is minimized. The value of the prediction error is obtained as:

$$
E=\sum_{P} E(w)=\sum_{P} \sum_{i}\left(r_{i}^{P}-O_{i}^{P, i}\right)
$$

where $\mathrm{p}, O_{i}^{P, i}$, and $r_{i}^{P}$ indicate the quantity of the training data, the $\mathrm{i}^{\text {th }}$ neuron which belongs to the $\mathrm{l}^{\text {th }}$ output layer, and the $\mathrm{i}^{\text {th }}$ real output corresponding to the $\mathrm{p}^{\text {th }}$ training data, respectively. 
Based on Eq. (14) and Eq.(15), BP algorithm is used to transfer the bias terms and also the weight's terms:

$\omega_{i, j}^{i-1, l}(k+1)=\omega_{i, j}^{i-1, l}(k)-\lambda \frac{\partial E}{\partial \omega_{i, j}^{i-1, j}}$

$b_{j}^{l}(k+1)=b_{j}^{l}(k)-\lambda \frac{\partial E}{\partial b_{i}^{l}}$

Here, $\lambda$ indicates the learning rate, and $\mathrm{k}$ states the iteration numbers.

\subsection{RBF-NN}

The process of the RBF-ANNs is demonstrated in Fig. 3. It composed of 3 layers of input, hidden layers, and output [33].

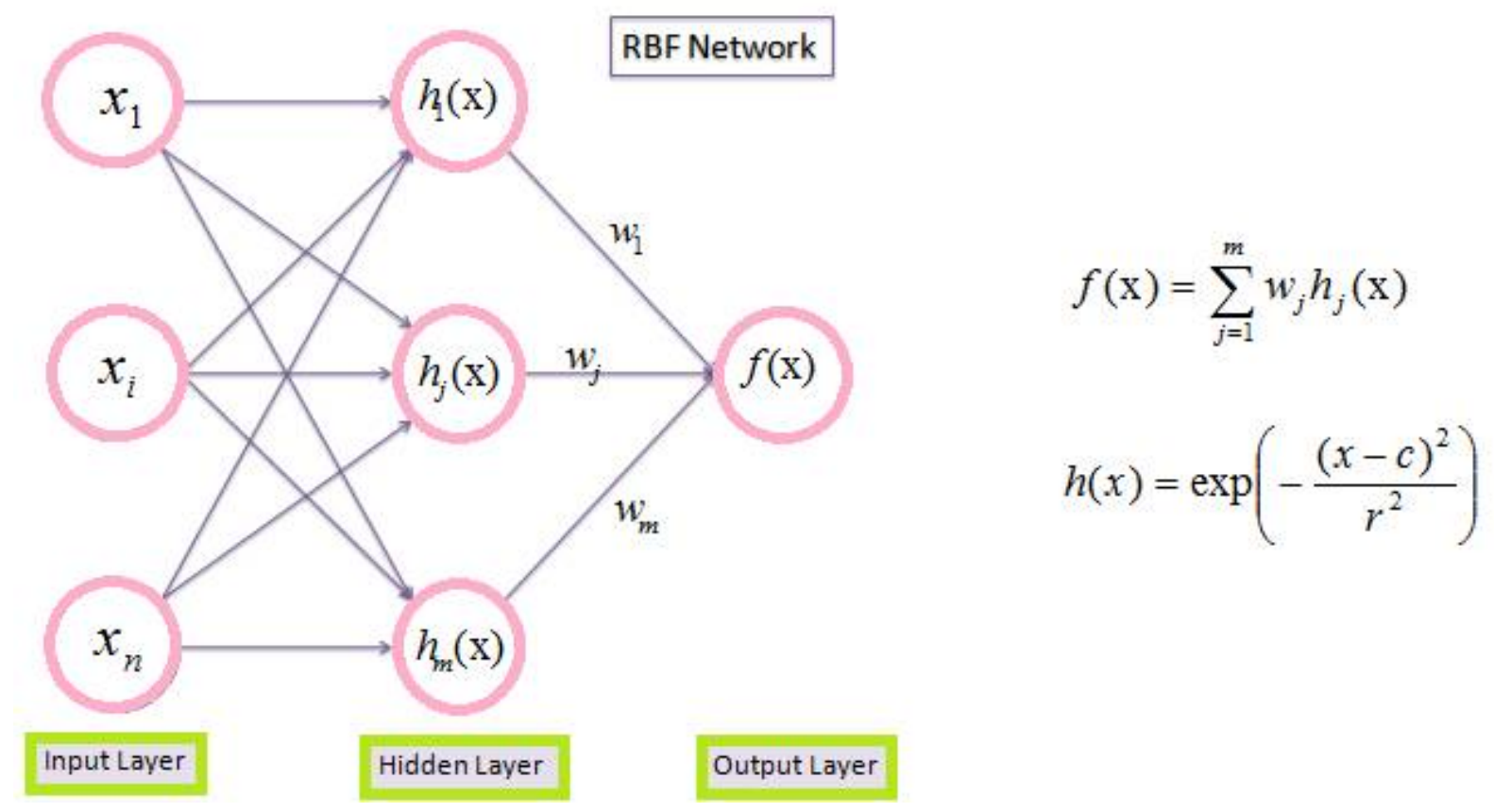

Figure 3: Construction of RBF-ANN approach

The MLP is structurally analogous to RBF-NNs. However, the calculation process is not similar since in the RBF-NNs, one hidden layer is existed and use and estimation in the calculation 
process but the MLP-NNs employ multiple hidden layers which are interconnected. Before applying the RBF-NNS, an activation function of the hidden layer must be defined and the highest quantity of the neurons should be specified. Here, neurons are considered as a process unit of the network. In addition, the assessment of the optimum values is the important task in the process to modify the process based on the assessment. Weights factor are used to train the RBF-NNs [34].

The major traits of the RBF-NNs are listed as follows:

- Triple layer structure.

- Activation functions of Gaussian are typically used in the hidden layer.

- Weight are delivered to the hidden layer and then assigned to the output layer.

- Acceptable degree of interpolation.

In the interpolation algorithm, the input data is mapped to the corresponding objective value of $t^{p}$. Thus, each input vector required an activation function. This process is performed by $\phi\left(\left\|x-x^{p}\right\|\right)$. Here, $\phi$ is the activation function and $\left\|x-x^{p}\right\|$ denotes the Euclidean position difference between $x$ and $x^{p}$. The output is calculated as follows:

$f(x)=\sum_{p=i}^{N} w_{p} \phi\left(\left\|x^{q}-x^{p}\right\|\right)=t^{p}$

where, $w_{p}$ is the weight factor and $x^{q}$ denotes the $\mathrm{q}^{\text {th }}$ input vector. In other words, to regulate the weight terms to come close to the Eq. (17), the interpolation process is necessary:

$f\left(x^{q}\right)=\sum_{p=i}^{N} w_{p} \phi\left(\left\|x^{q}-x^{p}\right\|\right)=t^{p}$

Among available activation functions, the Gaussian activation function is mostly used. This function is defined as follows:

$\phi(r)=\exp \left(-\frac{r^{2}}{2 \sigma^{2}}\right)$ 
where, $\sigma$ and $\mathrm{r}$ denote the interpolating function and the distance between a center of "c" and the local position of data point "x", respectively.

\subsection{LSSVM model}

Support Vector Machine (SVM) is considered as a noteworthy tool since its wide practicality in various cases. SVM has several features including wider converge to find the precious optimum, no further network regulation is required, lower regulation parameters, and more flexibility in overfitting issues. The following function can be considered for the SVM theory [35-38]:

$f(x)=w^{T} \emptyset(x)+b$

$\phi(\mathrm{x})$ and $W^{T}$ substitute the kernel function and the output layer vector, respectively. Furthermore, $\mathrm{b}$ and $\mathrm{x}$ represent the bias, and the inputs into the $N \times n$ matrix respectively. In this matrix the $\mathrm{N}$ denotes the trained data and $\mathrm{n}$ states the input parameters' number. Vapnik presented a meticulous procedure to obtain the weight and bias. In this process, the following function must be minimized [39]:

Objective Function $=\frac{1}{2} w^{T}+c \sum_{i=1}^{n}\left(\xi_{i}-\xi_{i}^{*}\right)$

By these following restrictions:

$$
\left\{\begin{array}{c}
y_{k}-w^{T} \emptyset\left(x_{k}\right)-b \leq \varepsilon+\xi_{k}, k=1,2, \ldots, N \\
w^{T} \emptyset\left(x_{k}\right)+b-y_{k} \leq \varepsilon+\xi_{k}{ }^{*}, k=1,2, \ldots, N \\
\xi_{k}, \xi_{k}{ }^{*} \geq 0
\end{array}\right.
$$

In above equations, $x_{k}$ is the $\mathrm{k}^{\text {th }}$ input, $y_{k}$ indicates the $\mathrm{k}^{\text {th }}$ output, $\varepsilon$ indicates the accuracy of the function estimation, $\xi_{k}$ and $\xi_{k}^{*}$ denote the slack factors. In overall, in order to specify the allowable 
deviations, slack terms are employed. A modifiable term of $\mathrm{c}>0$ requires to adjust the value range of the deviation from the $\varepsilon$.

SVM method is modified to Least Square Support Vector Machine (LSSVM) to be able to cover linear equations through linear programing to get a faster and more curious response than the conventional SVM approach. The LSSVM approach is as follow:

Objective Function $=\frac{1}{2} w^{T} w+\frac{1}{2} \gamma \sum_{k=1}^{N} e_{k}^{2}$

While: $\quad y_{k}=w^{T} \emptyset\left(x_{k}\right)+b+e_{k}$

In above equations, the training parameter is denoted by $\gamma$ and the regression error of the training steps is represented by $e_{k}$.

Moreover, in comparison to the SVM method, equality constraints are used instead of the inequality constraints. The Lagrangian approach is used to solve the above problem (Eq. (22) and Eq. (23)):

$L(w, b, e, a)=\frac{1}{2} w^{T} w+\frac{1}{2} \gamma \sum_{k=1}^{N} e_{k}^{2}-\sum_{k=1}^{N} a_{k}\left(w^{T} \emptyset\left(x_{k}\right)+b-e_{k}-y_{k}\right)$

here, $a_{k}$ indicates the Lagrangian multipliers and its derivatives should be equal to zero for solving the process. Furthermore, the following equations of Eq. (25) should be employed:

$\left\{\begin{array}{c}w=\sum_{k=1}^{N} a_{k} \emptyset\left(x_{k}\right) \\ \sum_{k=1}^{N} a_{k}=0 \\ a_{k}=\gamma e_{k}, k=1,2, \ldots, N \\ y_{k}=w^{T} \emptyset\left(x_{k}\right)+b+e_{k}, k=1,2, \ldots, N\end{array}\right\}$

Therefore, the LSSVM method should be applied to solve the $2 \mathrm{~N}+2$ equations and other unknown variables of $e_{k}, a_{k}, \mathrm{w}$, and $\mathrm{b}$. 
$\gamma$ indicates the regulating variable of the LSSVM approach. Since the both of SVM and LSSVM methods are used kernel functions, the presence of another tuning parameters is vital. Here, RBF kernel has been employed:

$k\left(x, x_{k}\right)=\exp \left(\frac{-\left\|x_{k}-x\right\|^{2}}{\sigma^{2}}\right)$

$\sigma^{2}$ is act as a regulating parameter. Therefore, the target parameters of the LSSVM can be obtained more precisely by decreasing the error between the predicted results and the actual illustrations. For LSSVM approach; the mean square error (MSE) is presented as follows:

$M S E=\frac{\sum_{i=1}^{n}\left(\alpha_{i, e x p}-\alpha_{i, p r e d}\right)^{2}}{n}$

$\mathrm{n}$ denotes to the quantity of the primary population, $\alpha$ states the output amount of $\mathrm{CO}_{2}$. The subscript Pred. stands for predicted data points and exp. is the experimental data points.

Here, the Suykens and Vandewalle [35] LSSVM model is applied and the Genetic Algorithm (GA) is utilized in order to perform an optimization to regulate the parameters of the LSSVM model. The schematic diagram of the LSSVM technique is illustrated in Fig. 4. In this procedure, the data point are classified into 2 subclasses of training and testing in the first stage. The LSSVM network is composed based on the training data. $\sigma^{2}$ and $\gamma$ are arbitrarily guessed and then GA modified the values by means of minimizing the MSE between the real output and predicted value. This algorithm is performing continuously to obtain the desired objectives. 


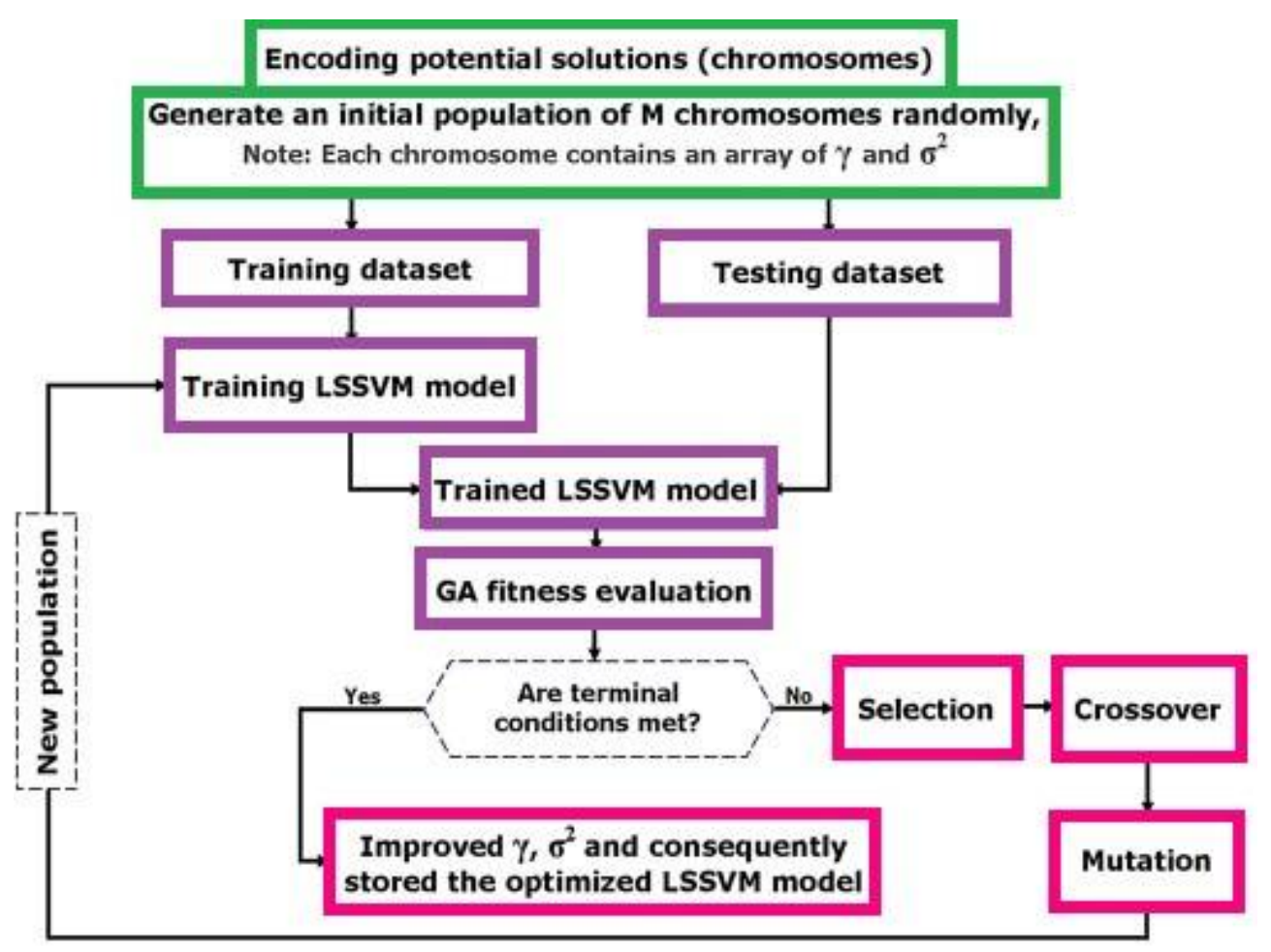

Figure 4: The LSSVM-GA model

\section{Experimental Procedure and Data Preparation}

In overall, PV/T is composed of two major sections: solar PV panel and thermal section. In this investigation, a polycrystalline PV panel (i.e. $90 \mathrm{~W}$, area: $0.73 \mathrm{~m}^{2}$ ) is provided for experimental measurments. The aosorber plate of the thermal section is consists of a serpentine tube connected to an aluminum sheet with $3 \mathrm{~mm}$ thickness( See Figure 5). 


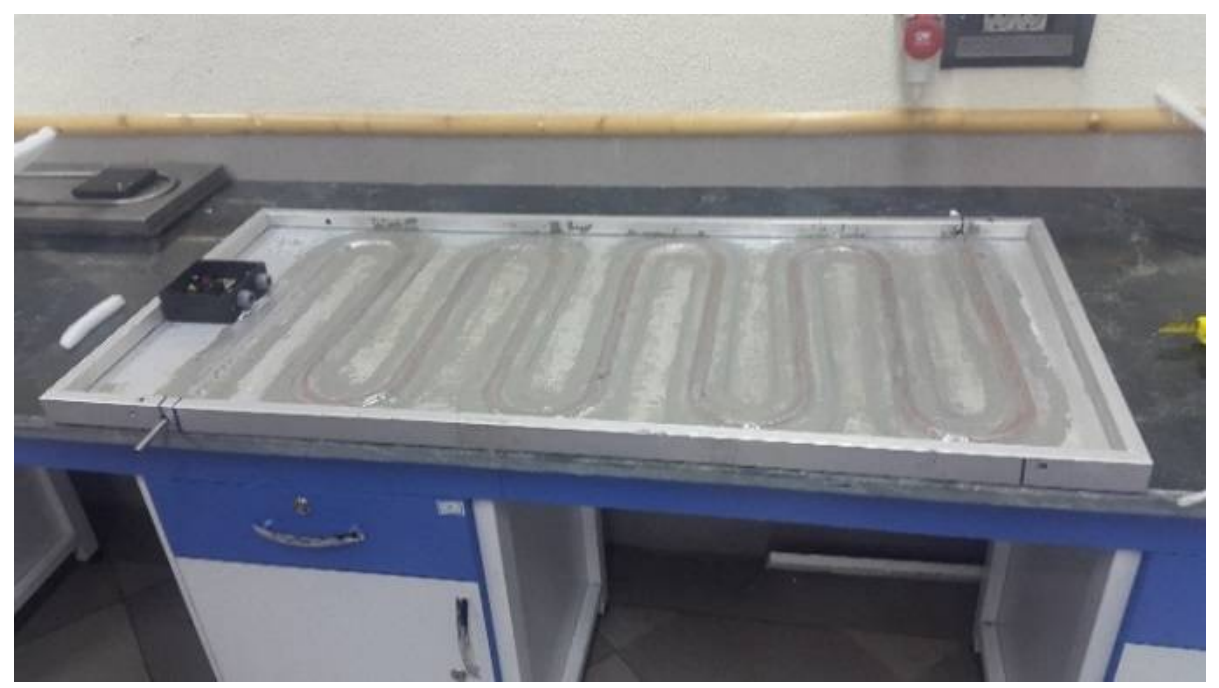

Figure 5. The PVT plate after connecting half-pipe

A 100 liters water tank for transfering the heat form the PV/T system is then provided and adjoined to the PV panel. In addition, the water circulation process is done bu an electric water pump. The whole setup is demonstrated in Figure 6. 


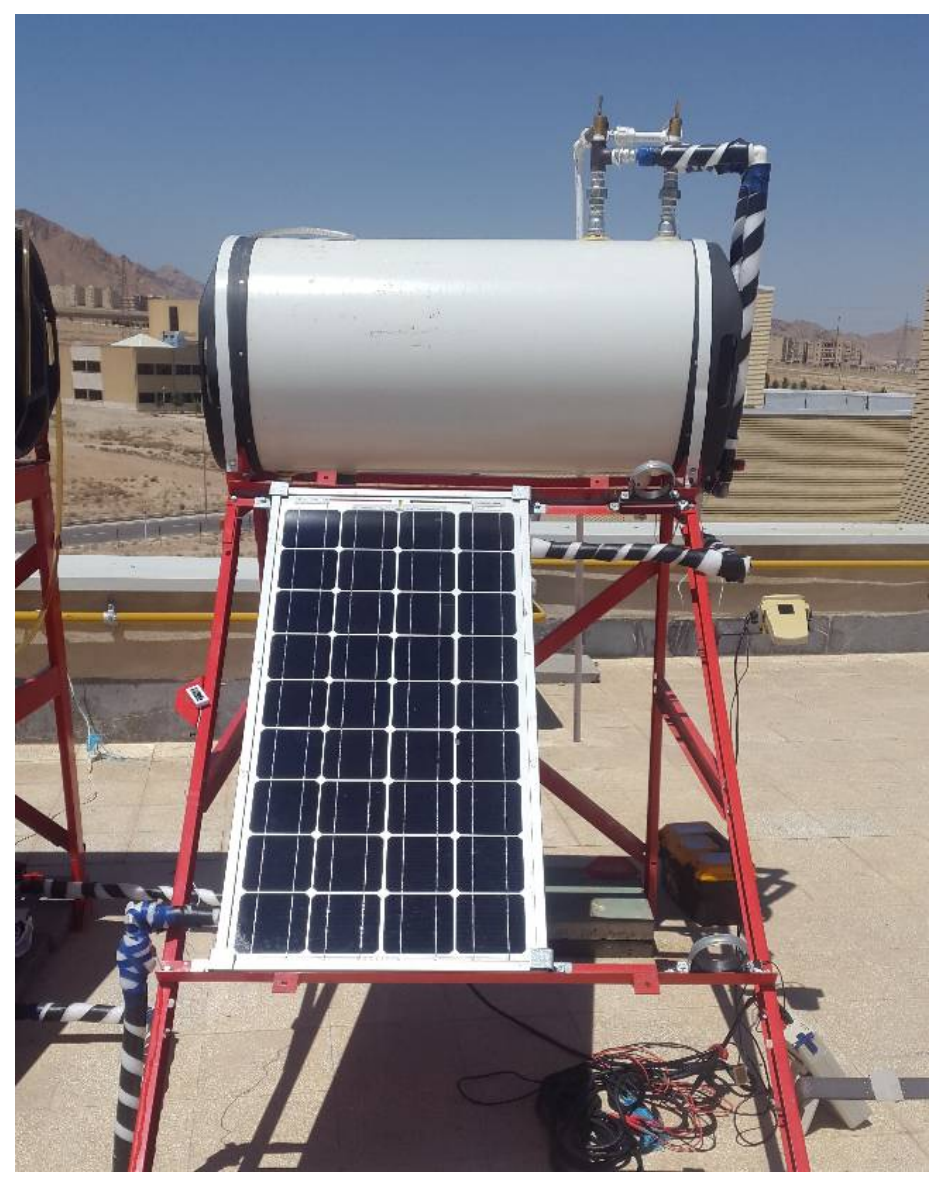

Figure 6. PVT system during test

Data logging were done by a flowmeter to regulate the circulation flow rate, Pyranometer to recroed the solar irradiation, solar power analyzer to recored the operational statuses of the PV 
panel, and also three thermometers at inout, midpoint, and output of the tank to measure the water tempreature.

The system was experimented in a sunny summer day almost in the noon to have constant and maximum amount of solar irradiations. The vaiations of the solar irradiance during the tests in different days is illistrated in Figure 7.

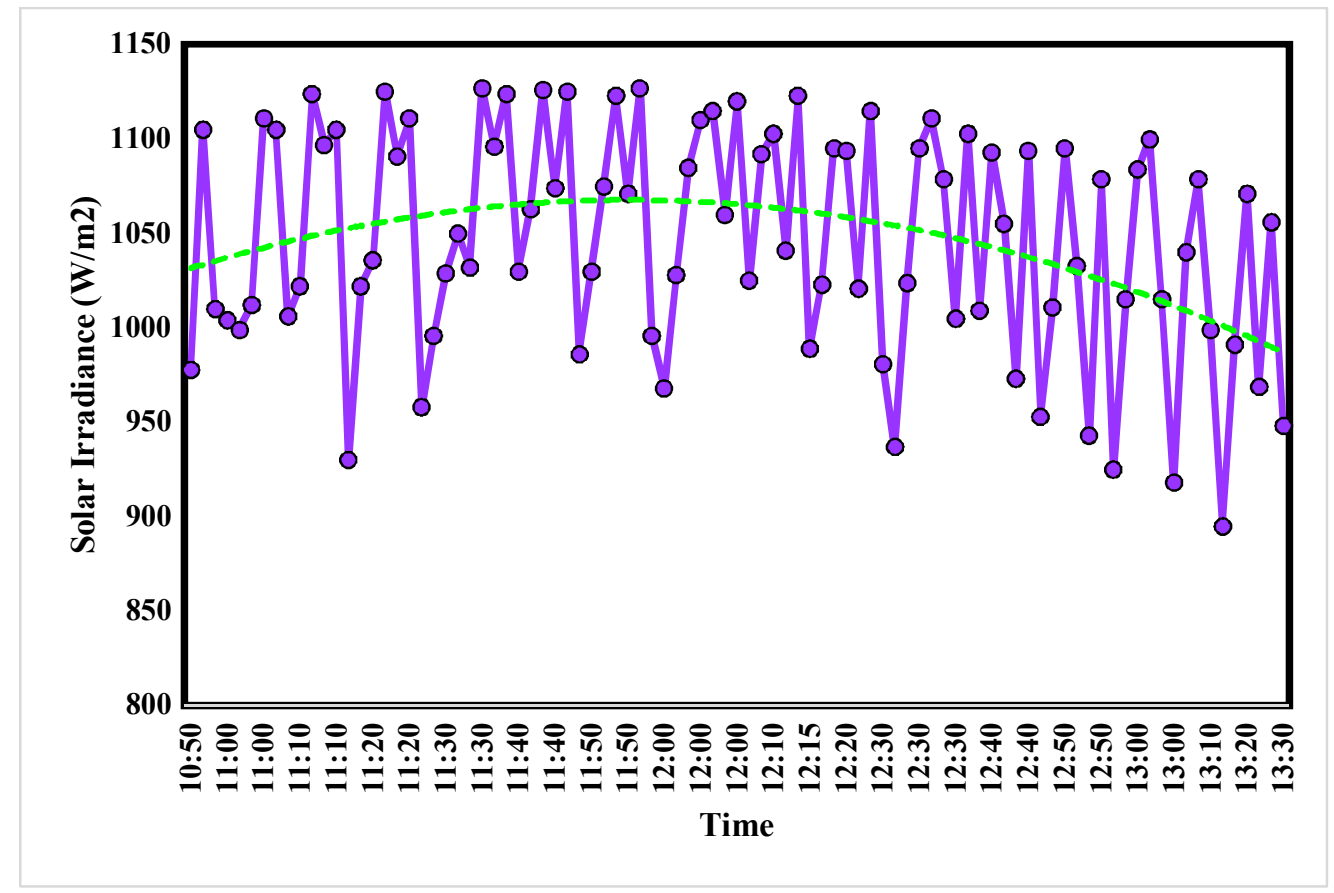

Figure 7. Data set for solar irradiance

Water mass flow rate is an essential factor in the PV/T system. Here, in this study, the water mass flow rate is $\frac{1}{2}$ to $4 \mathrm{lit} / \mathrm{min}$ and other system parameters are recorded. In addition, the influence of water inlet temperature $\left(20^{\circ} \mathrm{C}<\mathrm{T}_{\text {inlet }}<45^{\circ} \mathrm{C}\right)$ on the PV/T system is experimented.

Fig. 8 demonstrates the parallel diagram of affecting parameters and their ranges at various heat of sun on the PV/T system. Fig. 9 illustrates Andrews diagram of all parameters to have a visual insight from high-dimensional data. This diagram is a non-integer model of the Kent-Kiviat radar diagram or the smoothened model of a parallel coordinate diagram [40]. 


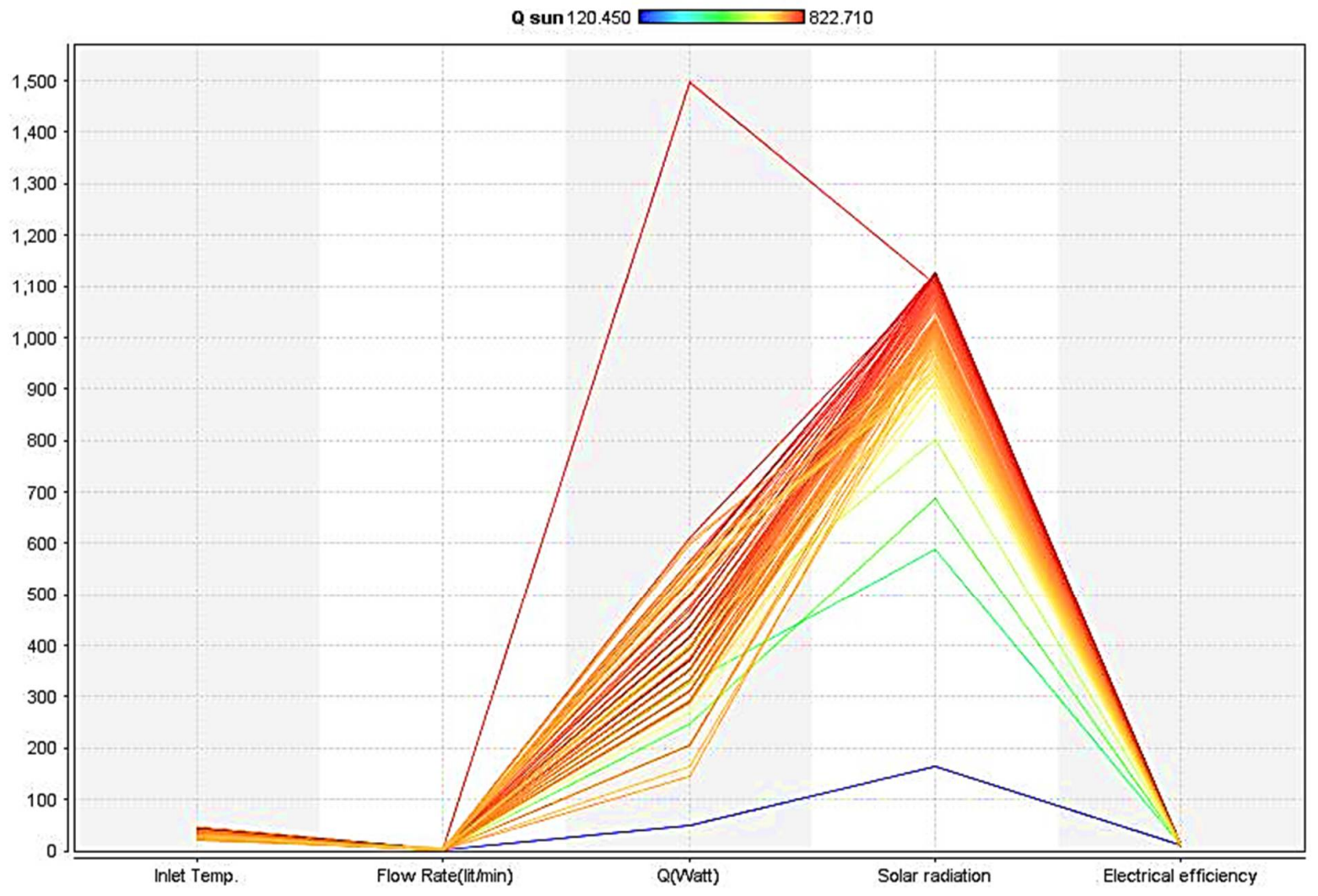

Figure 8: Parallel diagram of studied parameters in the present study of efficieny measurement of a $\mathrm{PV} / \mathrm{T}$ collector 


$$
\text { v }
$$



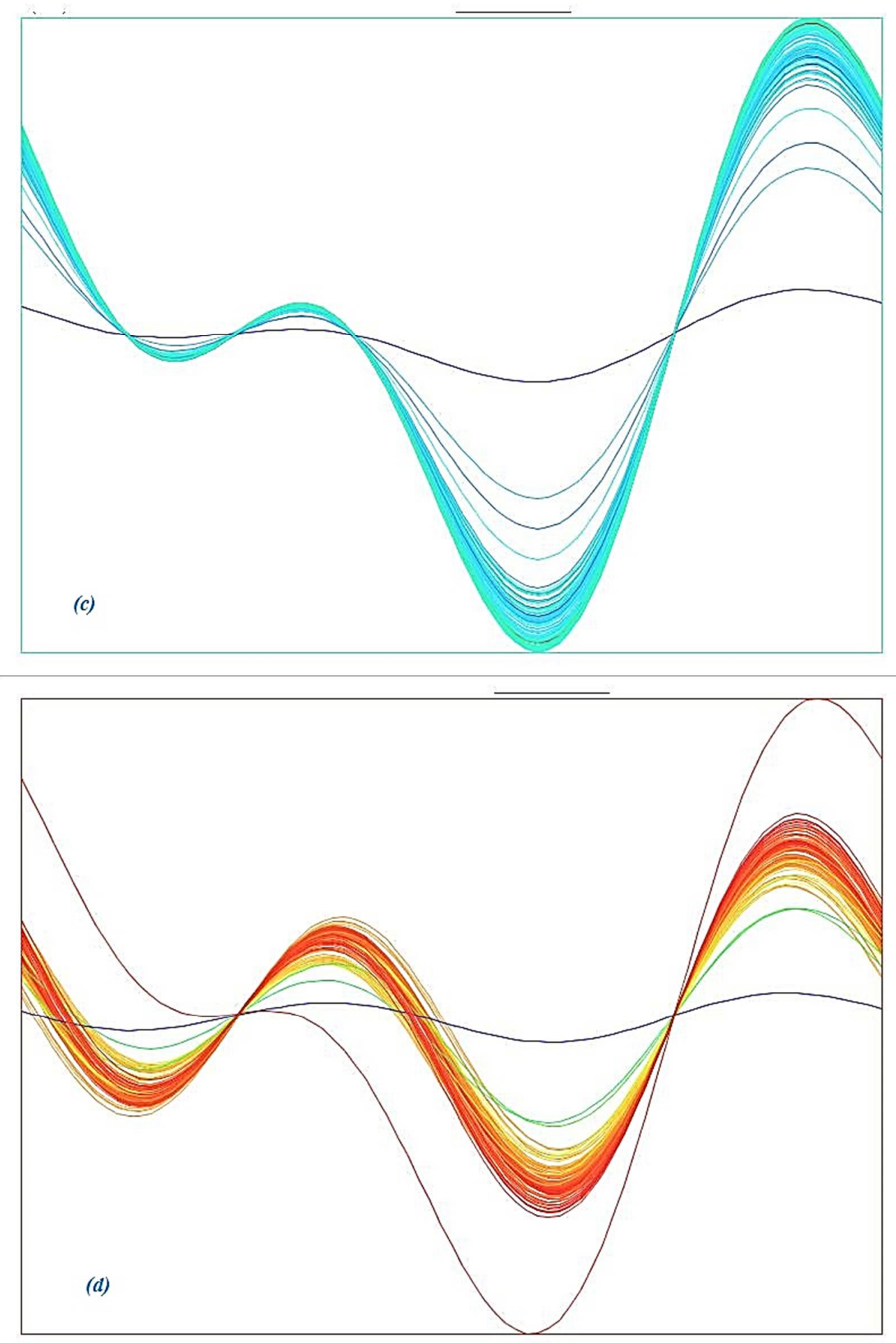

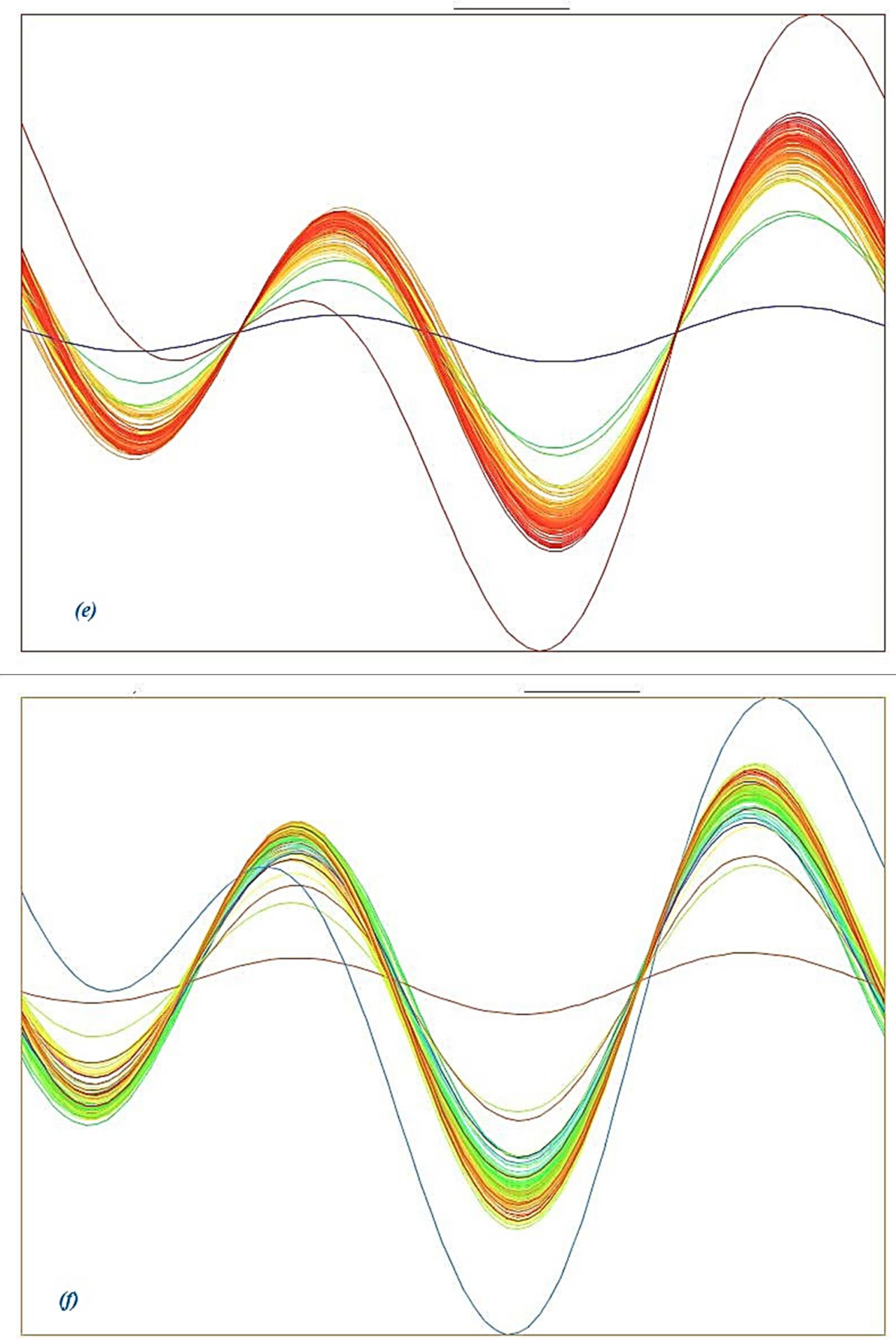

Figure 9: Andrew plots of (d) inlet temperature, (b) flow rate, (c) heat, (d) solar radiation, (e) heat of sun, and (f) electrical efficiency 
Moreover, a proper tool for evaluation of rough linear correlations of metadata is scatterplot matrices. For all of the applied parameters of this study, the scatter plot was drawn and illustrated in Fig. 10. In this figure, all of the parameters are placed diagonally. Each parameter is plotted against other parameters.
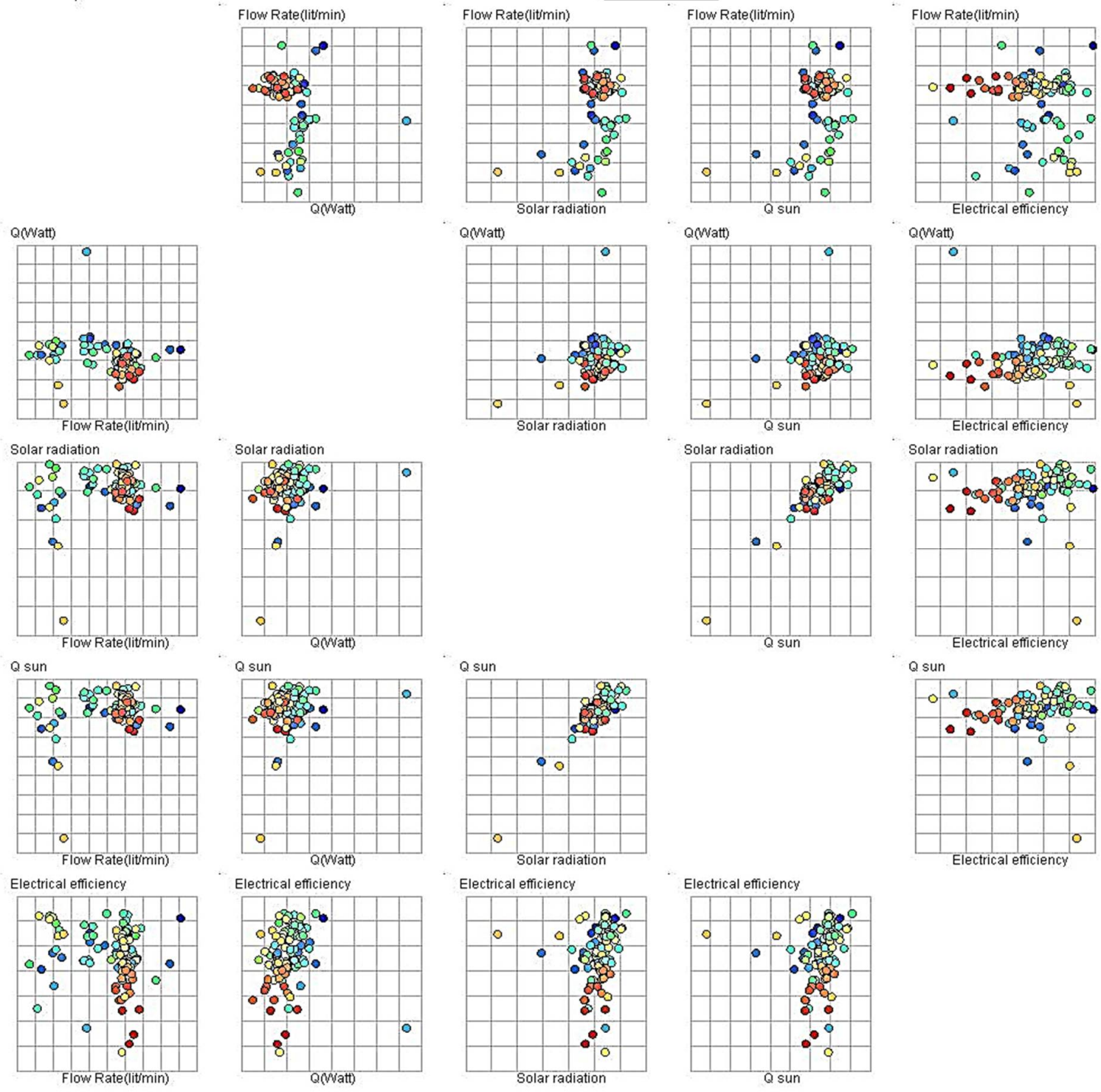
Figure 10: Scatter matrix plot for the studied parameters in the efficiency measurement of a $\mathrm{PV} / \mathrm{T}$ collector

\section{Models Implementation}

\subsection{Preprocessing Procedure}

Four machine-based prediction models of MLP-ANN, RBF-ANN, ANFIS, and LSSVM were developed in Matlab 2016 software to model the efficiency of the PV/T system. In order to find the objective of the efficiency of the PV/T system, some affecting parameters are assumed to be known and inserted as an input to the model. These variables are inlet temperature and flow rate of the flowing working fluid in the circuit of the collector, heat, solar radiation, and heat of sun. Overall number of 98 data were utilized in the aforementioned models to forecast the desired objective.

The data is classified into two subclasses of training and test which $78 \%$ of the data are considered as training and the remaining are belong to the test subclass. The former is used to specify the objective variables of the developed models while the latter checks the precision of the models output. To have a homogenized data set, the following equation, Eq.(28) is used to normalize the data points in the normalization range of $[-1,1]$ :

$D_{n}=2 \frac{D-D_{\min }}{D_{\max }-D_{\min }}-1$

$\mathrm{D}$ is the variable, $n$ stands for normalized, $\min$ refers to minimum, and $\max$ states the maximum amounts of the corresponding variable. In these models, inlet temperature, flow rate, heat, solar 
irradiance, and heat of sun are the input of the problem while the electrical efficiency is designed to be the target objective.

\subsection{Model Development}

\subsubsection{ANN}

In this study, two types of artificial neural network of RBF and multi-layer perceptron (MLP) were utilized to forecast the output of electrical efficiency of the PV/T system. Seven hidden neurons were used to for the training section in order to specify the target parameter by minimizing the distance of the forecasted and actual measured data. The Levenberg-Marquardt algorithm was used to optimize the process. The mean squared error of the obtained forecasted values from the MLP practice is depicted in Fig. 11. Moreover, Table 1 presents the optimum of the bias and weight.

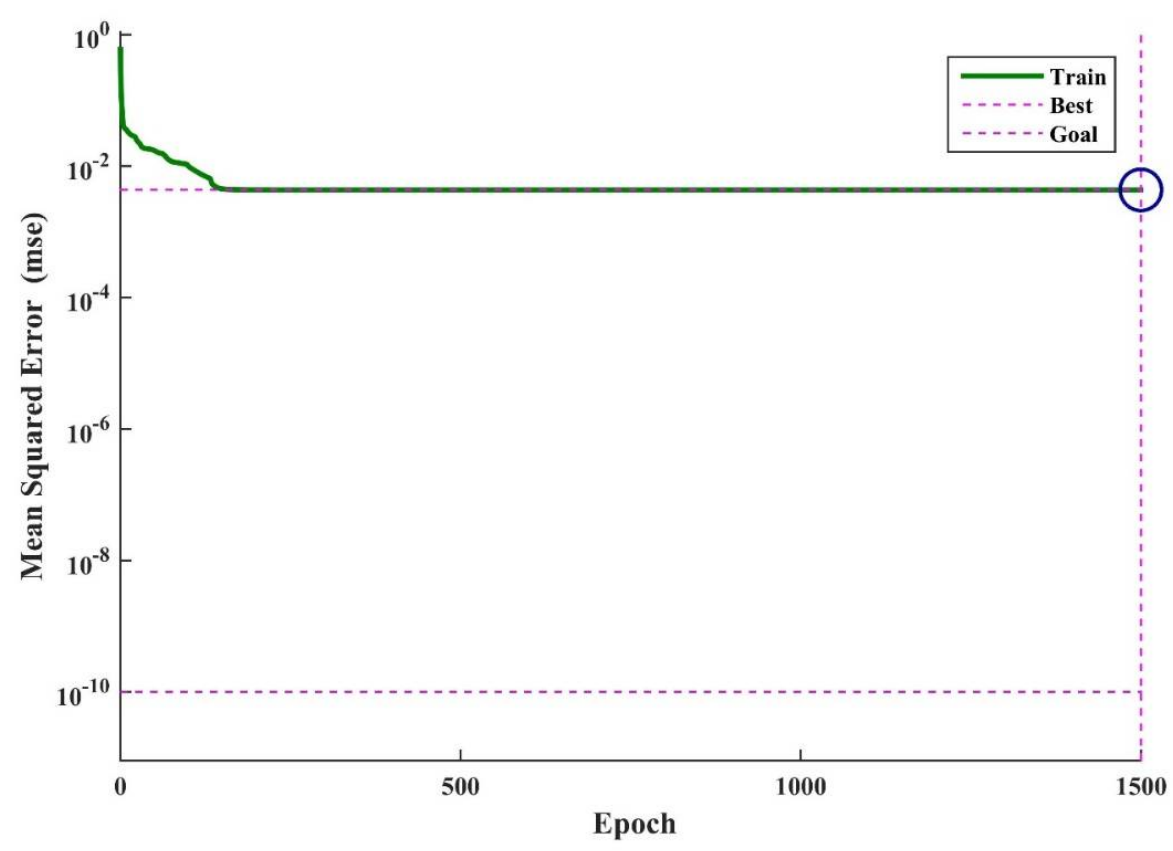

Figure 11: MLP-ANN performance during different iterations 
Table1: Optimum values of weight and bias in the MLP-ANN model

\begin{tabular}{|c|c|c|c|c|c|c|c|}
\hline \multicolumn{6}{|c|}{ Input layer } & \multicolumn{2}{|c|}{ Output layer } \\
\hline \multicolumn{5}{|c|}{ Weight } & Bias & Weight & Bias \\
\hline Inlet T & Flow & Heat & Solar & Heat of & b1 & Electrical & b2 \\
\hline & rate & & Rad. & Sun & & Eff. & \\
\hline-3.00217 & 14.62895 & 9.19011 & -6.14859 & -10.254 & 11.21392 & -0.77978 & 51.75337 \\
\hline 25.71744 & -43.7362 & -135.409 & 386.549 & 388.5208 & -811.641 & 1.63077 & \\
\hline 6.002147 & 30.00333 & -15.9983 & 5.146075 & 6.721965 & 4.233007 & -3.29637 & \\
\hline 0.022054 & -0.01171 & -0.01102 & -4.46868 & 4.423946 & -0.9196 & -166.279 & \\
\hline-77.2605 & 118.3238 & -20.377 & 72.74368 & 75.49725 & -152.927 & -1.55885 & \\
\hline 59.59531 & -54.9347 & 51.61157 & 15.50821 & 23.32499 & 5.853293 & -1.51761 & \\
\hline-12.356 & -25.3219 & 20.53642 & 0.310394 & 1.792913 & -11.9288 & -3.84421 & \\
\hline
\end{tabular}

In addition, to train the RBF-ANN model the Levenberg-Marquardt algorithm is used with 50 iterations. The training process of the radial basis network is regularly less time-consuming than the sigmoid or the linear network. The performance of the RBF-ANN method during various iterations is demonstrated in Fig. 12. 


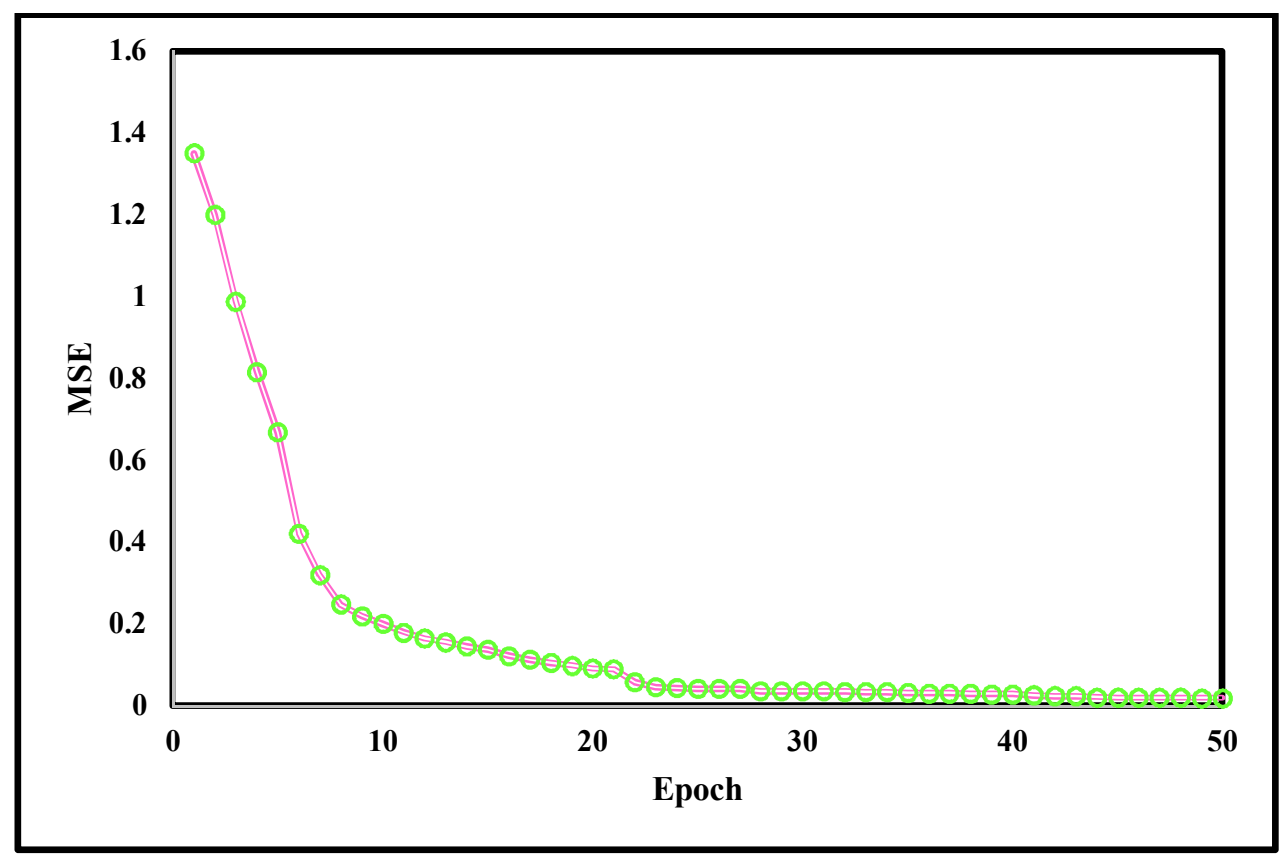

Figure 12: RBF-ANN performance during different iterations

\subsubsection{ANFIS}

In facilitate the advancement of the ANFIS model, the Particle swarm optimization (PSO) approach was used. The overall numbers of ANFIS variables is dependent on clusters' number, $N_{c}$, variables' number, $N_{v}$, and the number of membership function variables $\left(N_{M F}\right)$ as follows:

$N_{T}=N_{c} \cdot N_{v} \cdot N_{M F}$

The membership function of this study is the Gaussian membership function. $\mathrm{Z}$ and $\sigma^{2}$ are the two membership function variables. The primary input parameters are the electrical efficiency, inlet temperature, flow rate, heat, solar radiation, and heat of sun. Seven clusters are primarily considered. Hence, the overall number of ANFIS parameters is 84 . In order to obtain the optimum status of the ANFIS parameters, the RMSE between experimentally measured and forecasted 
values is reflected as the cost function of the PSO algorithm Fig. 13. The RMSE of each iteration is shown. The trained membership function for input data is illustrated in Fig. 14.

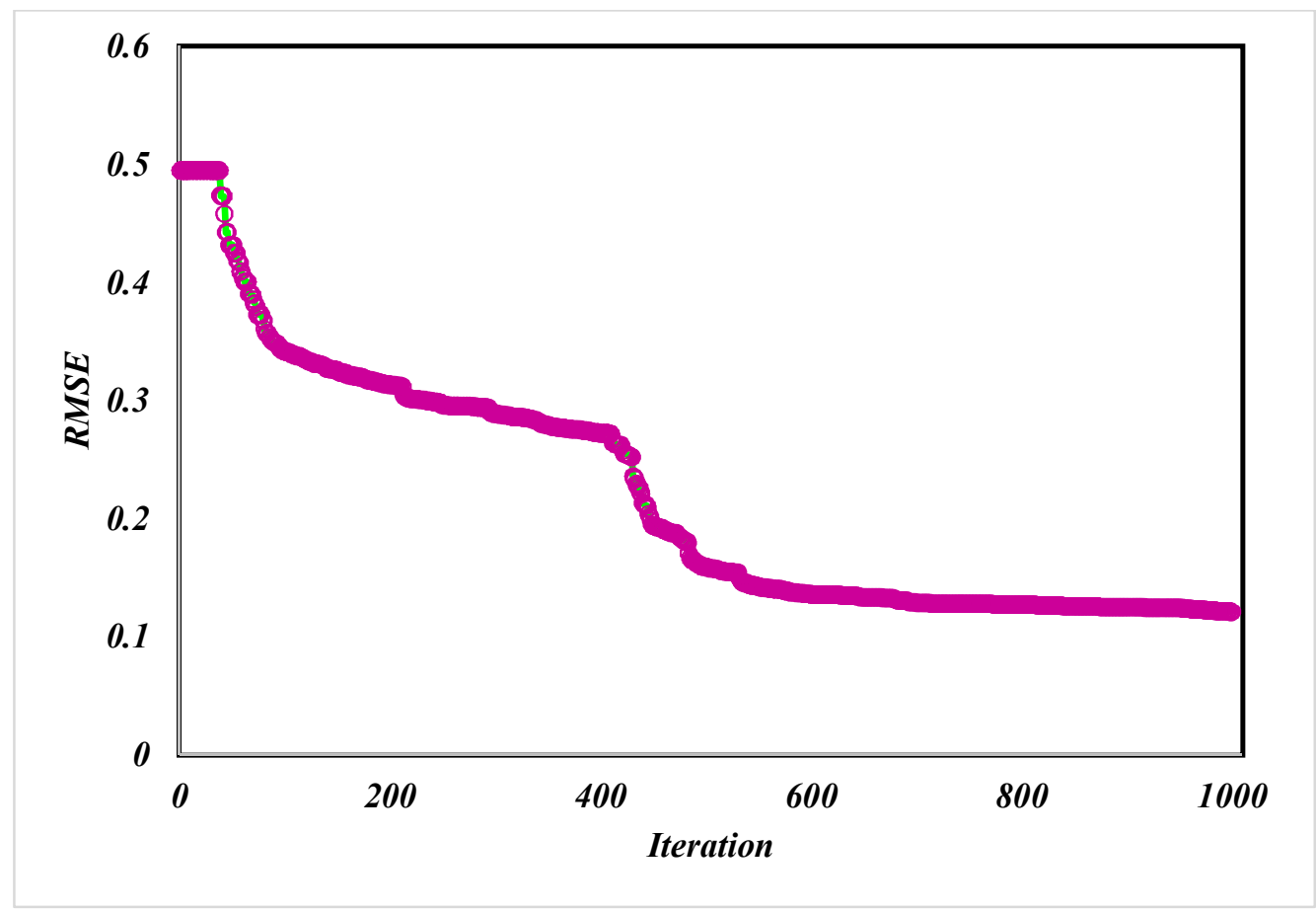

Figure 13: Applying PSO algorithm at various iterations in the training stage of the ANFIS model 


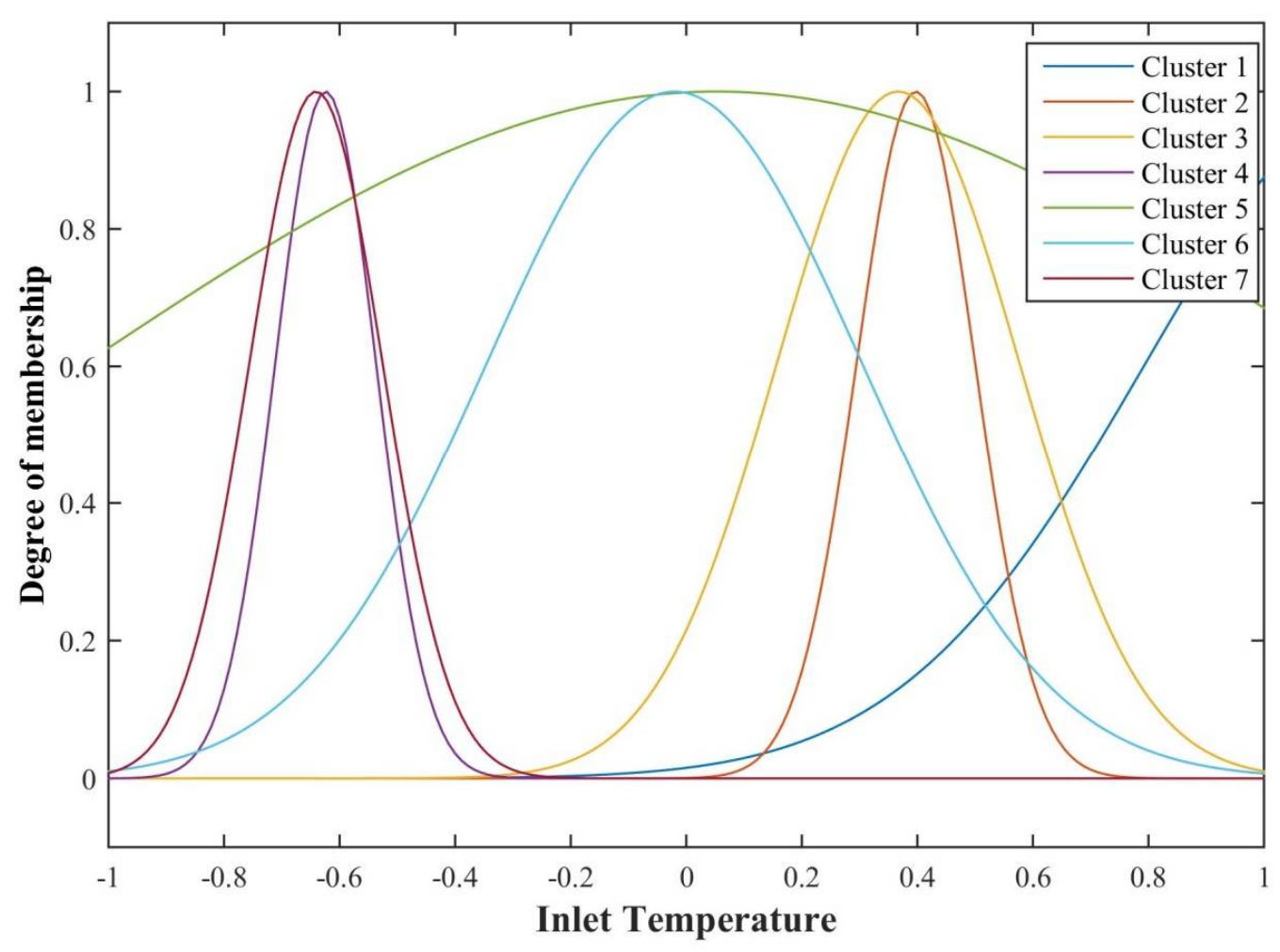



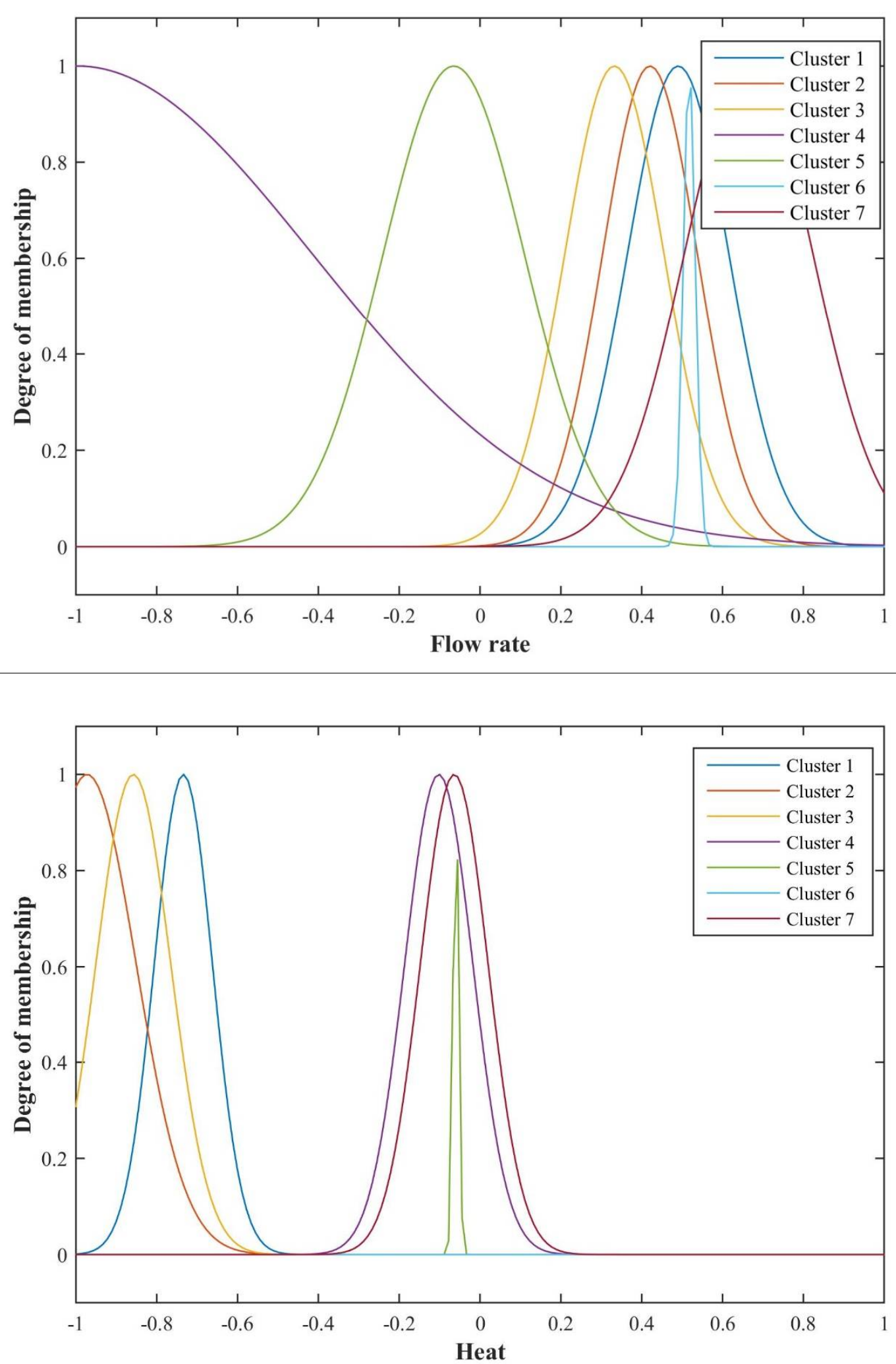

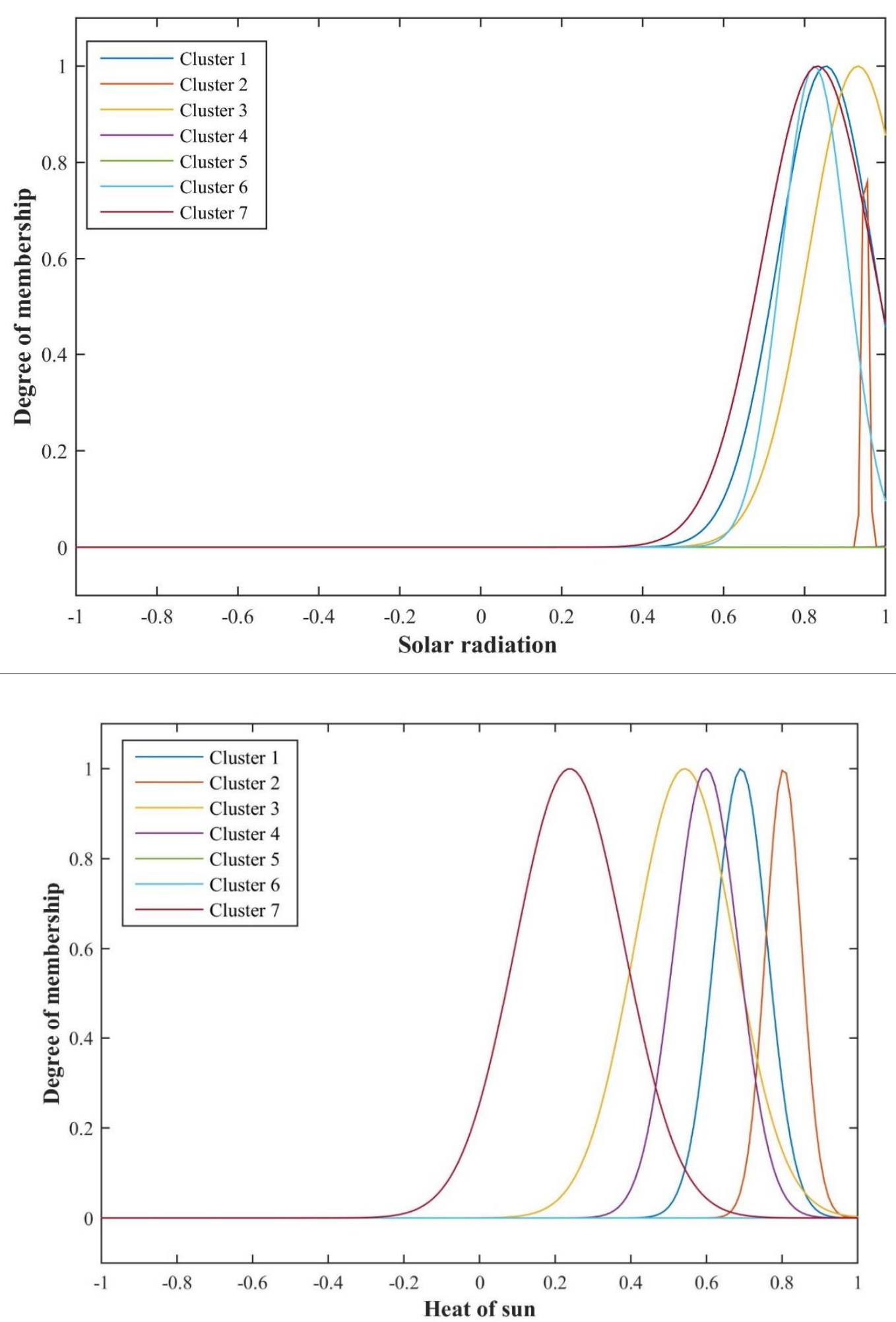

Figure 14: Fuzzy inference system for input variables: Training 


\subsubsection{Least squares support vector machine (LSSVM)}

The LSSVM approach employs two regulating variables in its algorithm. These variables are $\gamma$ and $\sigma^{2}$. The regulation variable is stated by $\gamma$ and the kernel variable is the RBF. In addition, the LSSVM method is also hybridized with GA to specify the optimum response of the introduced model.

\subsection{Models' Evaluation}

The proposed approaches are evaluated based on various statistical methods as listed in the following:

Mean Squared Error $(M S E)=\frac{1}{N} \sum_{i=1}^{N}\left(H_{i}^{\text {exp. }}-H_{i}^{\text {cal. }}\right)^{2}$

Average Relative Deviation $(A R D)(\%)=\frac{100}{N} \sum_{i=1}^{N} \frac{\left|H_{i}^{\text {exp. }}-H_{i}^{\text {cal. }}\right|}{H_{i}^{\text {exp. }}}$

Standard Deviation $(S T D)=\left(\frac{1}{N-1} \sum_{i=1}^{N}\left(H_{i}^{\text {exp. }}-H_{i}^{\text {cal. }}\right)^{2}\right)^{0.5}$

Root Mean Squared Error $(R M S E)=\left(\frac{1}{N} \sum_{i=1}^{N}\left(H_{i}^{\text {exp. }}-H_{i}^{c a l .}\right)^{2}\right)^{0.5}$

Correlation Coefficient $\left(R^{2}\right)=1-\frac{\sum_{i=1}^{N}\left(H_{i}^{\text {exp. }}-H_{i}^{\text {cal. }}\right)^{2}}{\sum_{i=1}^{N}\left(H_{i}^{\text {exp. }}-\bar{H}^{\text {exp }}\right)^{2}}$ 
where $N$ denotes the quantity of data points, the superscripts of exp. and cal. are for values which experimentally and based on calculation were obtained, respectively. $\bar{H}^{\text {exp }}$ indicates the mean efficiency obtained through experimental measurements.

\section{Results and Discussion}

The obtained results from applying four introduced intelligent techniques are described in detail in Table 2. The used data set consists of 98 data points.

Table 2. Models' charactristics and further information .

\begin{tabular}{|c|c|c|c|}
\hline \multicolumn{2}{|c|}{ LSSVM } & \multicolumn{2}{|c|}{ ANFIS } \\
\hline $\begin{array}{l}\text { Type } \\
\text { Kernel function }\end{array}$ & $\begin{array}{l}\text { Value } \\
\text { RBF }\end{array}$ & $\begin{array}{l}\text { Type } \\
\text { Membership Function }\end{array}$ & $\begin{array}{l}\text { Value/comment } \\
\text { Gaussian }\end{array}$ \\
\hline$\Gamma$ & 6942.0845 & No. of MF parameters & 84 \\
\hline$\sigma^{2}$ & 8.01234 & No. of clusters & 7 \\
\hline Quantity of training data & 74 & Quantity of training data & 74 \\
\hline Quantity of testing data & 24 & Quantity of testing data & 24 \\
\hline Population size & 100 & Population size & 50 \\
\hline Iteration & 1000 & Iteration & 1000 \\
\hline $\mathrm{C}_{1}$ & 1 & $\mathrm{C}_{1}$ & 1 \\
\hline $\mathrm{C}_{2}$ & 2 & $\mathrm{C}_{2}$ & 2 \\
\hline \multicolumn{2}{|c|}{$M L P-A N N$} & \multicolumn{2}{|c|}{$R B F-A N N$} \\
\hline Type & Value/comment & Type & Value/comment \\
\hline $\begin{array}{l}\text { Quantity of input } \\
\text { neuron layer }\end{array}$ & 5 & Quantity of input neuron layer & 5 \\
\hline $\begin{array}{l}\text { Quantity hidden } \\
\text { neuron layer }\end{array}$ & 7 & Quantity of hidden neuron layer & 50 \\
\hline $\begin{array}{l}\text { Quantity of output } \\
\text { neuron layer }\end{array}$ & 1 & Quantity of output neuron layer & 1 \\
\hline $\begin{array}{l}\text { Hidden layer activation } \\
\text { function }\end{array}$ & Logsig & Optimization method & Levenberg-Marquardt \\
\hline $\begin{array}{l}\text { Output layer activation } \\
\text { function }\end{array}$ & Purelin & Quantity of training data used & 74 \\
\hline Optimization method & Levenberg-Marquardt & Quantity of testing data & 24 \\
\hline $\begin{array}{l}\text { Quantity of training } \\
\text { data for }\end{array}$ & 74 & Quantity of max iterations & 50 \\
\hline Quantity of testing data & 24 & & \\
\hline $\begin{array}{l}\text { Quantity of max } \\
\text { iterations }\end{array}$ & 1500 & & \\
\hline
\end{tabular}


Fig. 15 demonstrates the experimental plot for all investigated models, simultaneously. As it is monitored, all of the methods shows an acceptable agreement with the trend of experimental values. However, LSSVM approach is more precise based on the less deviation from experimental values in comparison to other methods. 


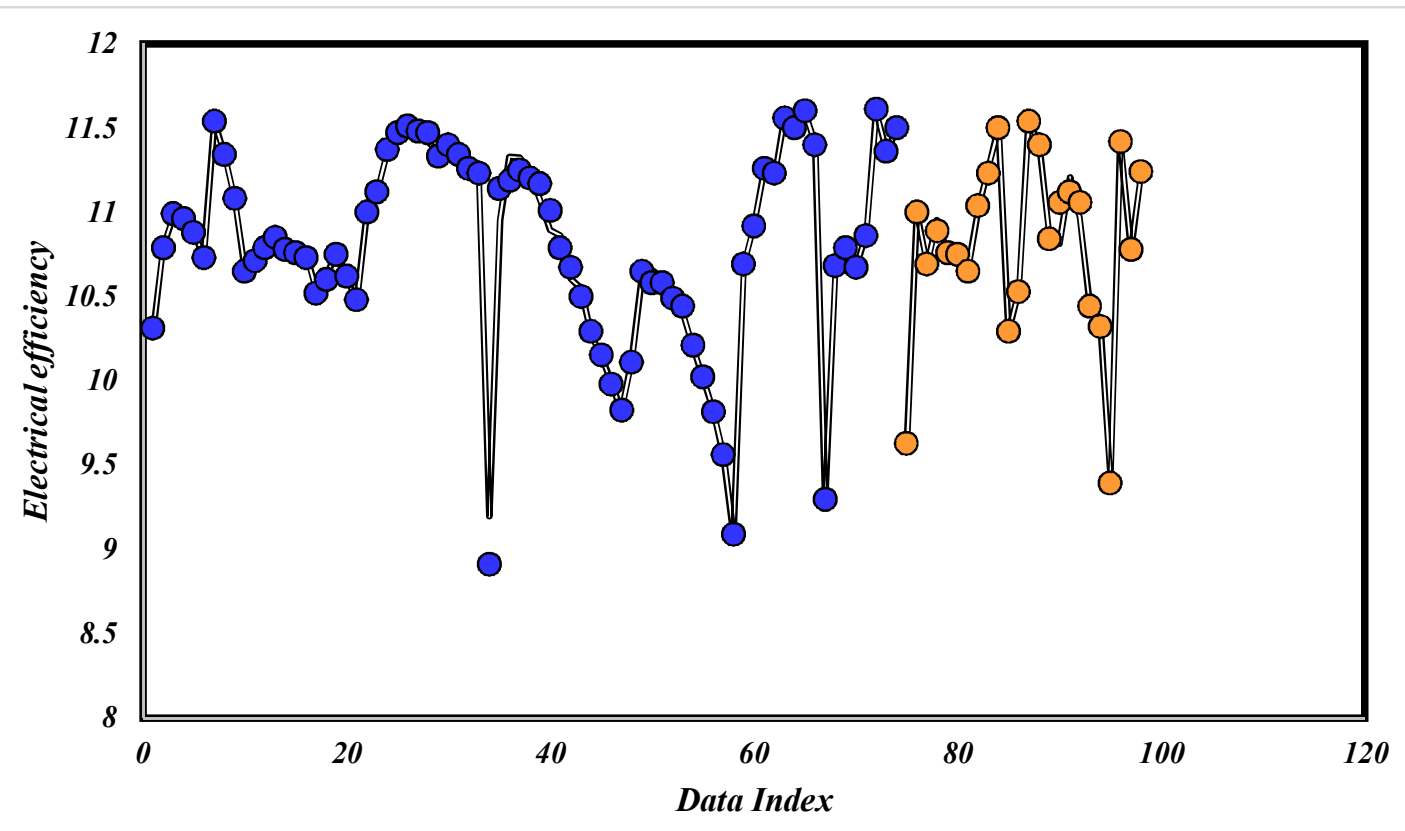

- Train Exp. O Test Exp. $\quad$ Train LSSVM $\rightleftharpoons$ Test LSSVM

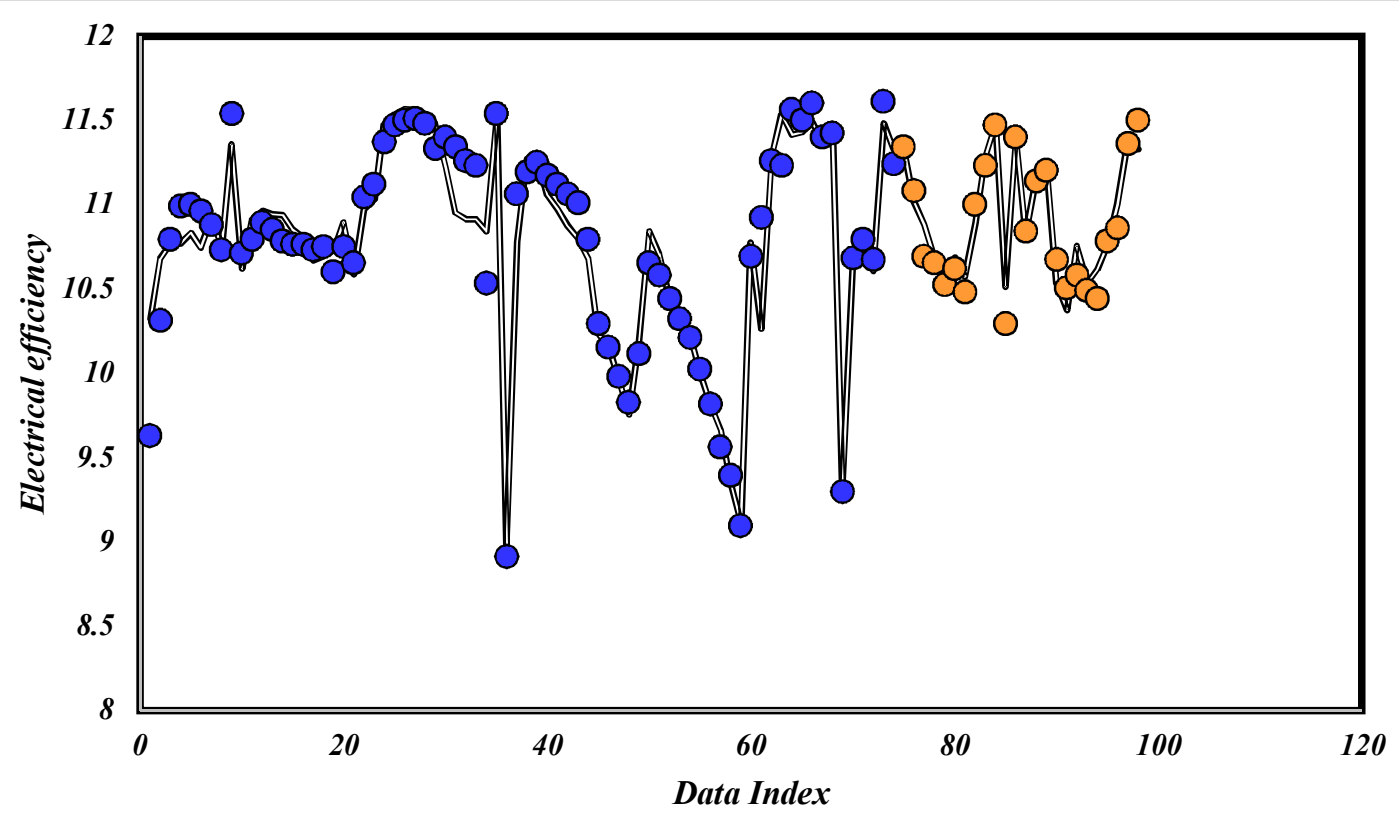

- Train Exp. O Test Exp. $\quad$ Train ANFIS 


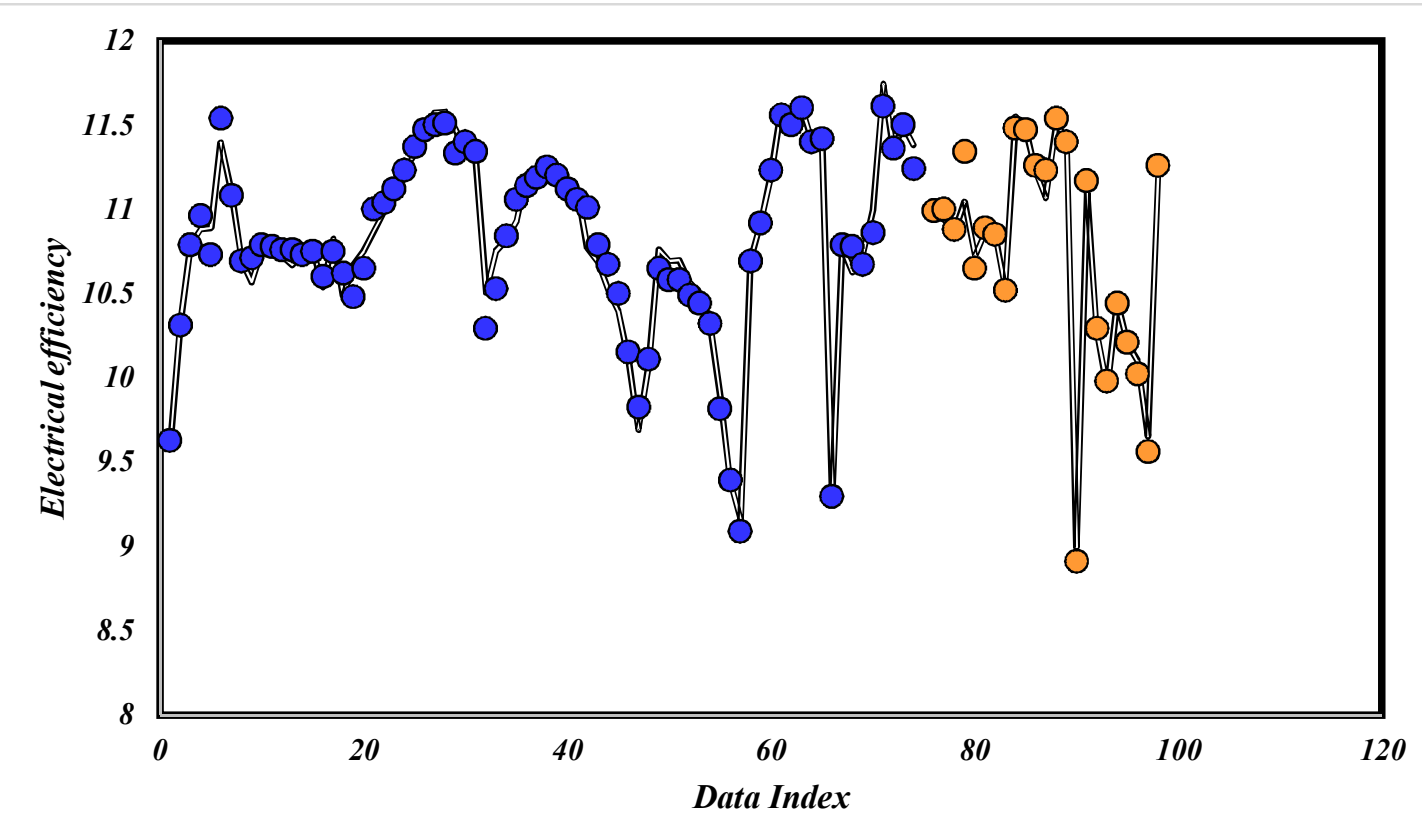

○ Train Exp. $\quad$ Test Exp. $\quad$ Train MLP-ANN $=$ Test MLP-ANN

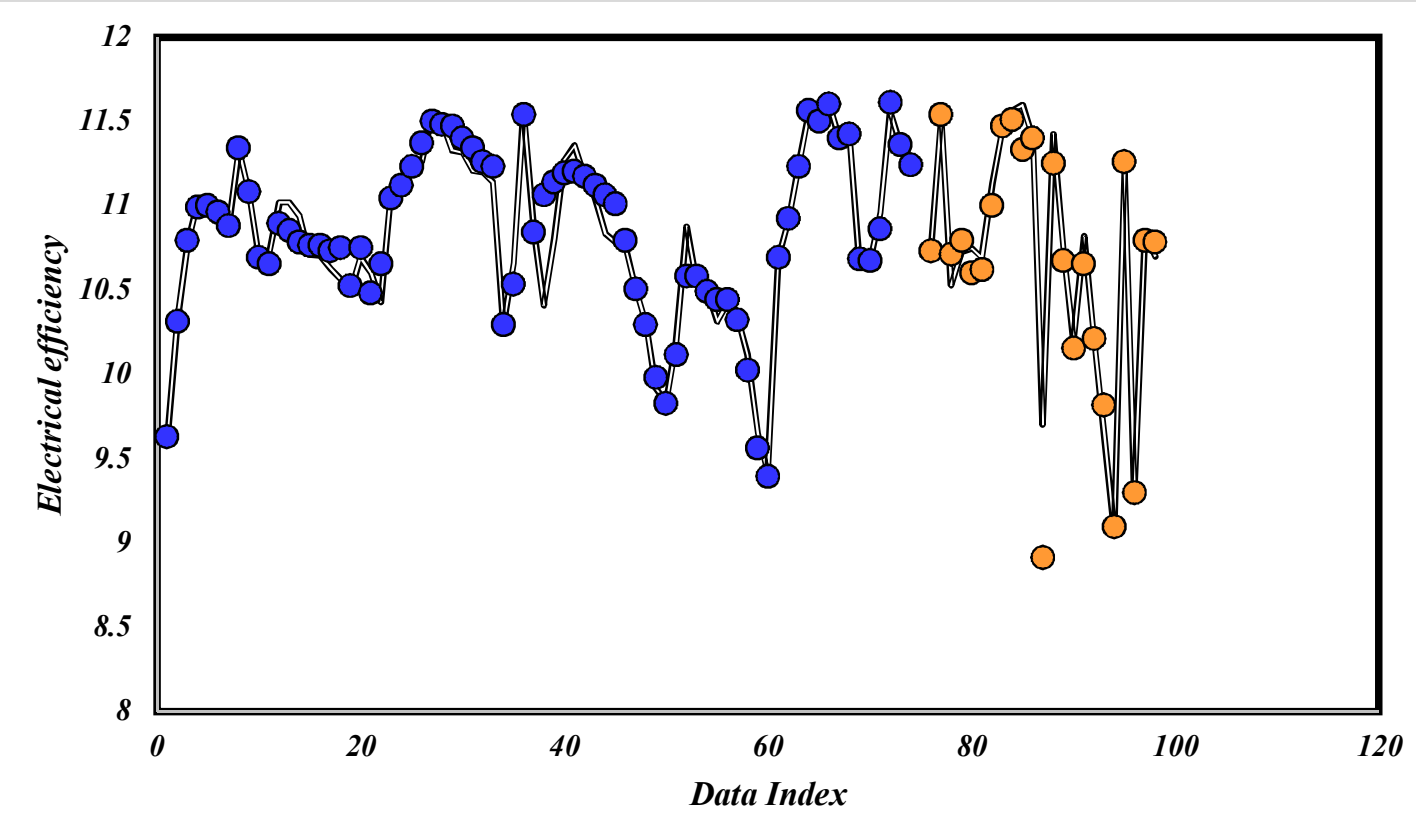

○ Train Exp. O Test Exp. $\quad$ Train RBF-ANN $\rightleftharpoons$ Test RBF-ANN

Figure 15: Experimental versus predicted electrical efficiency value 
Fig. 16 demonstrates the regression plot of the forecasted and experimentally measured values for the studied models. Based on this evaluation, it seems that most of the data are placed close to the $\mathrm{X}=\mathrm{Y}$ line. Fig.s 16 (a)-(c) illustrate the optimum fitting lines by using linear regression of experimentally measured data and forecasted values by machine-based methods. 

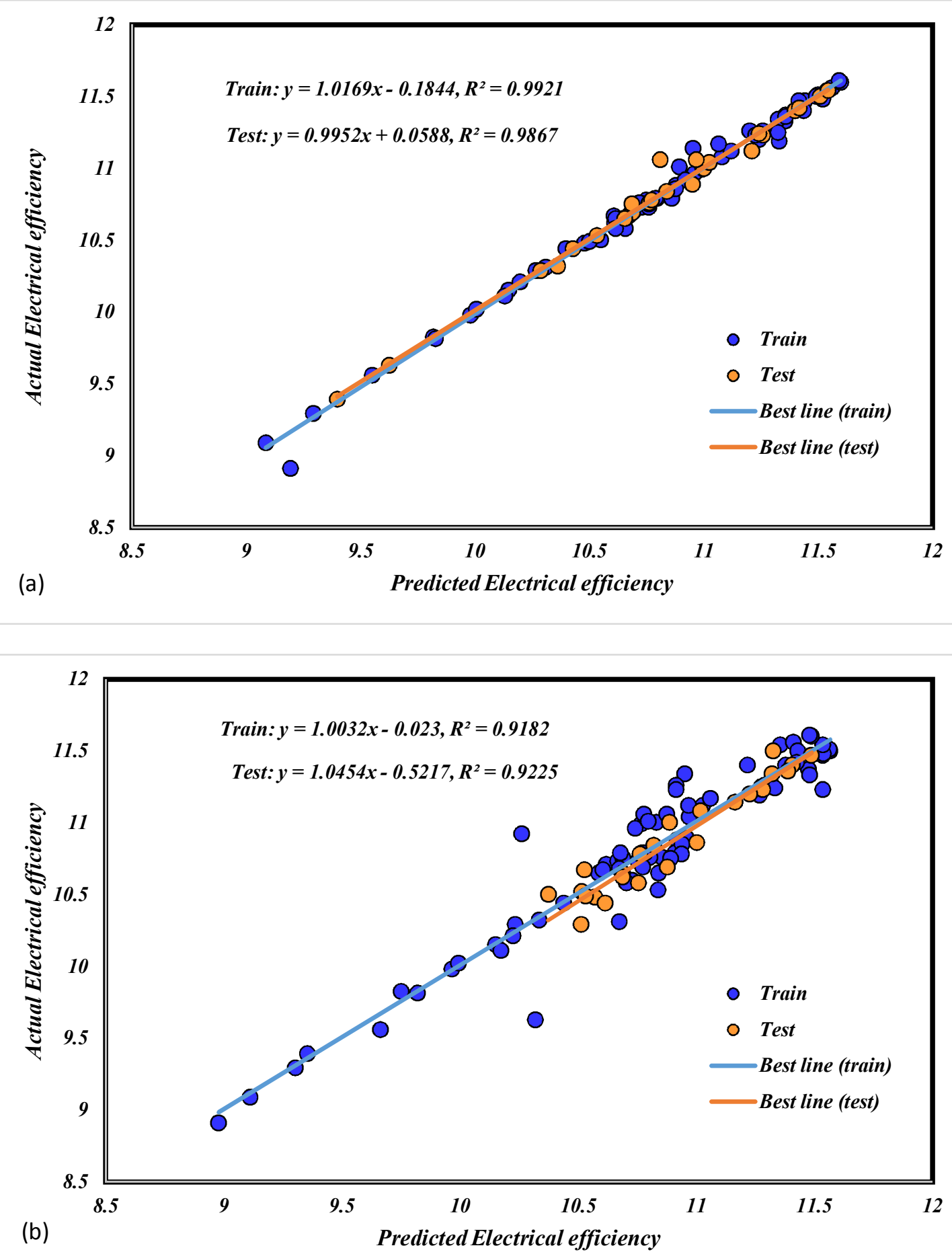

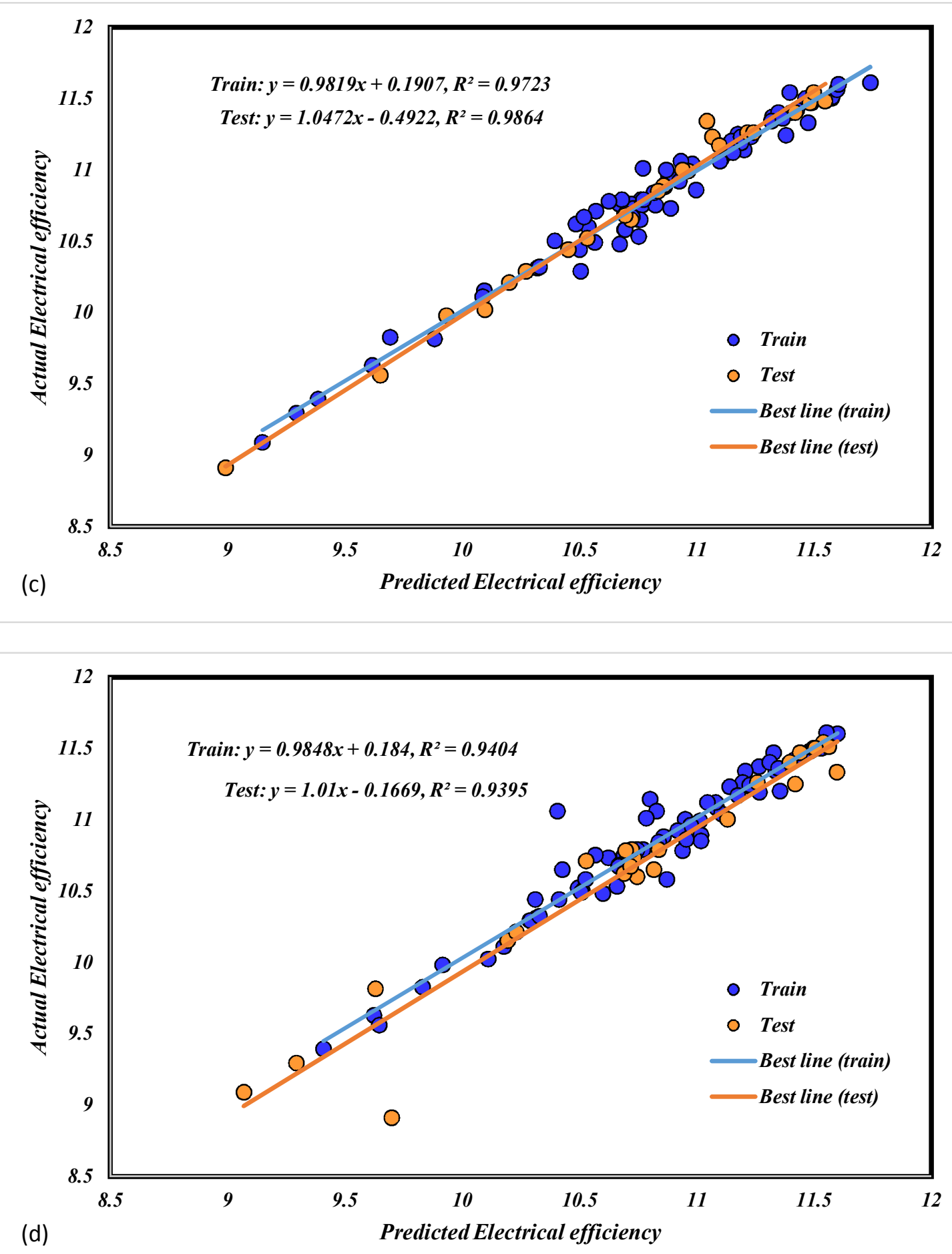

Figure 16: Regression plot of efficiency:experimental Vs. estimated (a) LSSVM, (b) ANFIS, (c) MLP-ANN, (d) RBF-ANN 
The deviation graph is another typical evaluation graph which is used to compare the valued of forecasted efficiency of the PV/T collector with the actual data resulted from the experiments. Fig. 17 depicts the deviation diagram for all of the introduced models. Based on the deviation plot, the closeness of the data near the zero line is higher in the LSSVM approach and therefore the lower deviation is resulted. The MLP-ANN, RBF-ANN, ANFIS, and LSSVM models resulted the values of $0.6,0.75,1.03$, and 0.27 for mean relative deviation of respectively. 

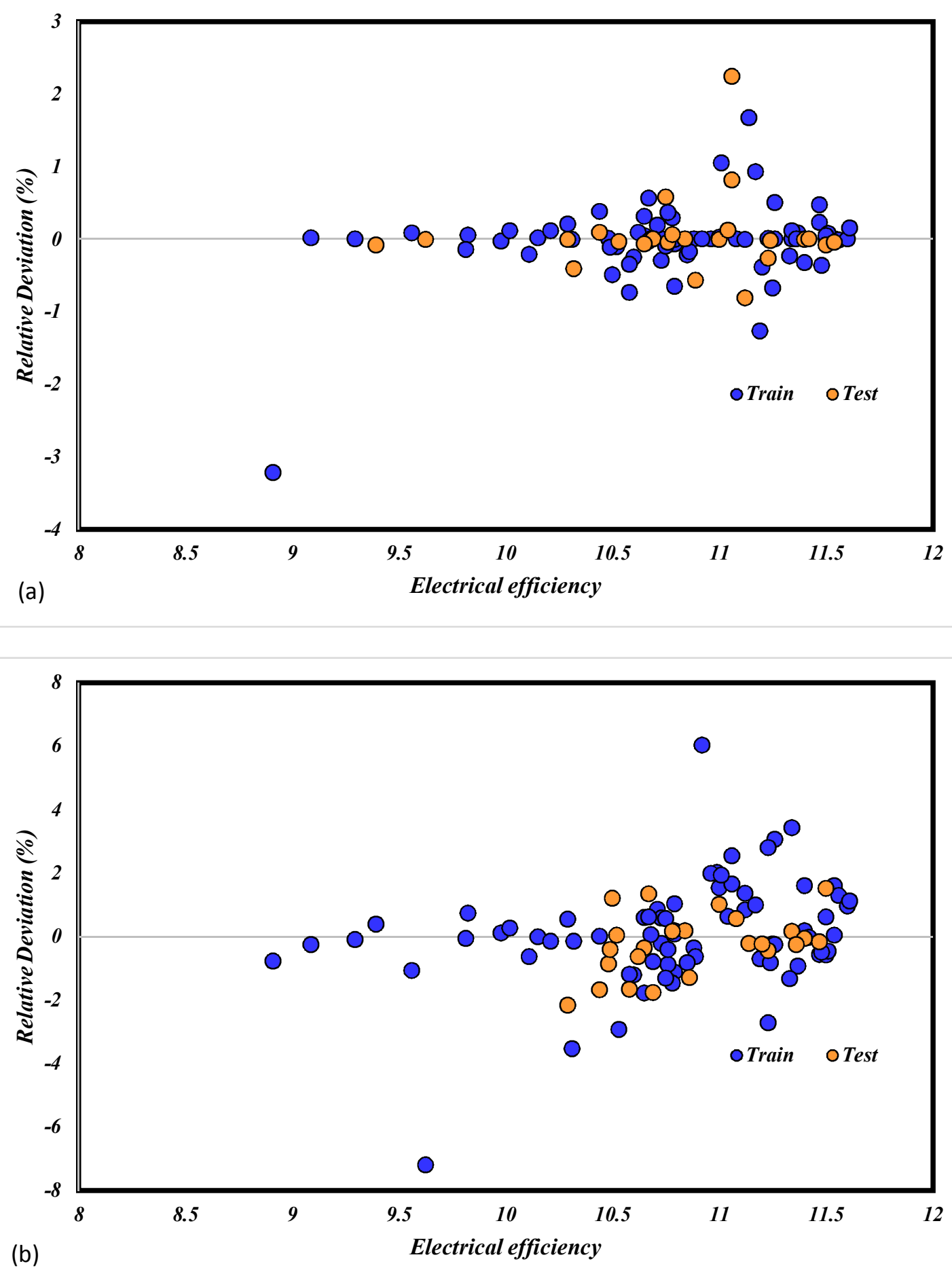

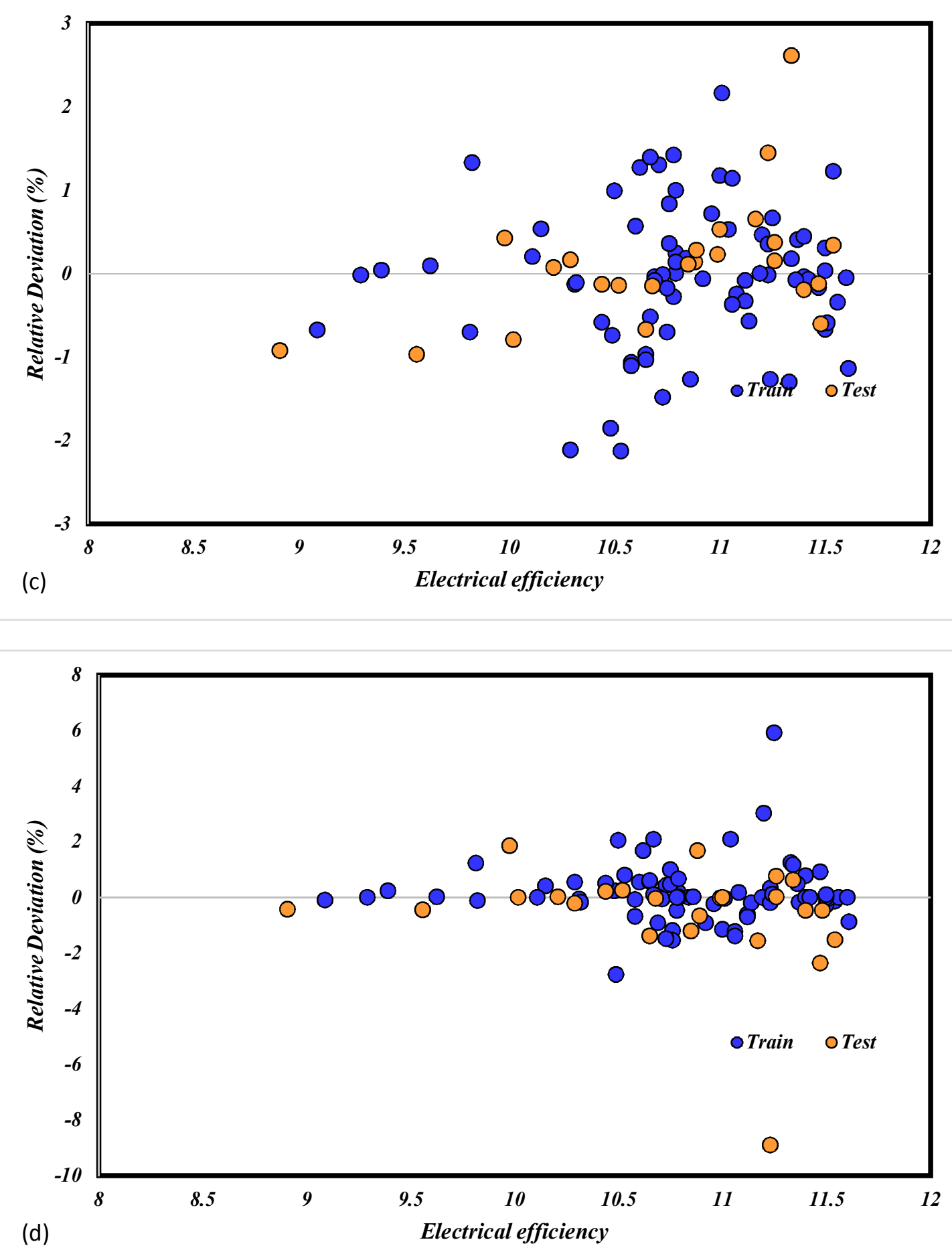

Fig. 17: Relative deviations of efficiency: experiment Vs. predicted (a) LSSVM, (b) ANFIS, (c) MLP-ANN, (d) RBF-ANN 
In order to examine the ability of the presented strategies, statistical error analyses are also carried out for train, test, and overall. Table 3. represents the results indicating that the proposed methods express precise estimation.

Table 3. Error analysis through different criteria.

\begin{tabular}{ccccccc}
\hline Model & & MSE & RMSE & MRE & $\mathbf{R}^{\mathbf{2}}$ & STD \\
\hline LSSVM & Test & 0.004 & 0.061 & 0.265 & 0.987 & 0.055 \\
& Train & 0.003 & 0.053 & 0.253 & 0.992 & 0.046 \\
\multirow{3}{*}{ ANFIS } & Total & 0.003 & 0.055 & 0.256 & 0.991 & 0.048 \\
& Test & 0.011 & 0.107 & 0.768 & 0.922 & 0.069 \\
& Train & 0.032 & 0.178 & 1.123 & 0.918 & 0.132 \\
MLP-ANN & Total & 0.027 & 0.164 & 1.036 & 0.918 & 0.120 \\
& Test & 0.007 & 0.083 & 0.509 & 0.986 & 0.063 \\
& Train & 0.008 & 0.091 & 0.634 & 0.972 & 0.061 \\
RBF-ANN & Total & 0.008 & 0.089 & 0.603 & 0.976 & 0.061 \\
& Test & 0.037 & 0.193 & 1.049 & 0.940 & 0.165 \\
& Train & 0.015 & 0.123 & 0.656 & 0.940 & 0.100 \\
& Total & 0.020 & 0.143 & 0.752 & 0.937 & 0.119 \\
\hline
\end{tabular}

\subsection{Outlier Detection}

Trustworthiness of the employed models is extremely dependent on the experimentally measured data points. [41]. Outliers are called to those data (individual or group) which their behaving trend is not following other data points. Therefore, one of the most momentous step in evolution of models is to detect and remove the outliers. Thus, to specify the outliers, the Leverage analysis by implementing standardized residuals $(\mathrm{R})$ is utilized. The outlying candidates are explored through drowing the William's plot, i.e. the graph of Rs against hat values $(\mathrm{H})$. The $\mathrm{H}$ are the diagonal arrays of the hat matrix and calculate as follows to specify the feasible space.

$H=X\left(X^{T} X\right)^{-1} X^{T}$ 
$X$ denotes a $[\ldots]_{n \times k}$ matrix where $n$ is the quantity of data and $k$ indicates the number of input variables. Feasible space is a squared region constrained to cut-off value on vertical axis and also limited to the warning leverage value on horizontal axis. Warning leverage is defined as:

$H^{*}=3 \frac{k+1}{n}$

$\mathrm{R}=3$ is the recommended cut-off value. The lines of $R= \pm 3$ on the vertical axis limit the feasible region. On the other hand, the feasible space on the horizontal axis is specified between lines of $H=0$ and $H=H^{*}=0.09$. Those data were outside of the acceptable range are called the Outlying. Based on the William's plot which is depicted in Fig. 18, most of the data are placed in the acceptable range except for one data for all studied models. 

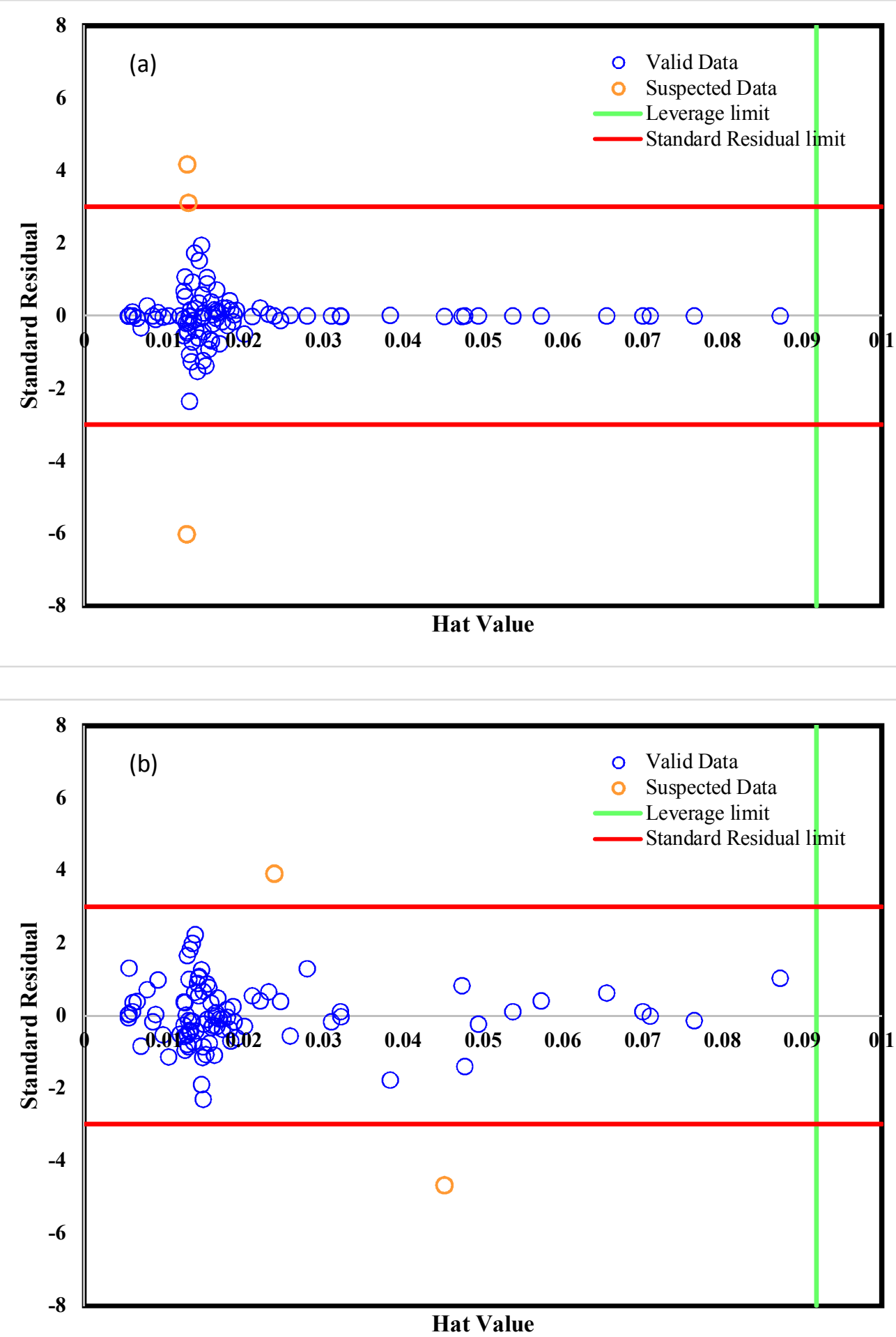

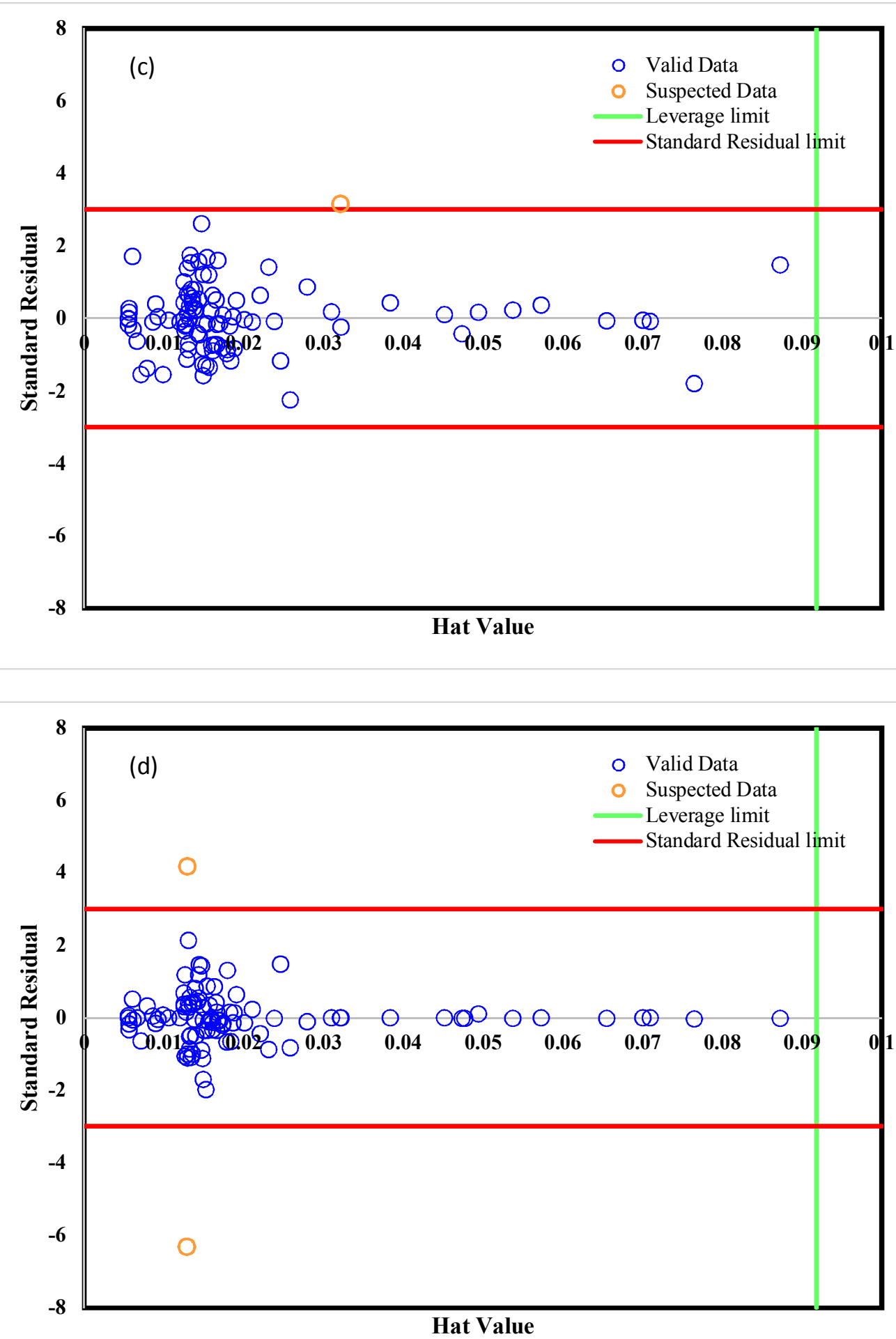

Fig. 18: William's plot for: (a) LSSVM, (b) ANFIS, (c) MLP-ANN, (d) RBF-ANN 


\subsection{Sensitivity Analysis}

In order to demonstrate the reliance of the objective of the study on input parameters a sensitivity analysis is carried out. A relevancy factor of $-1<\mathrm{r}<+1$ is selected in the sensitivity analysis. As the $\mathrm{r}$ is closer to unity states that the objective final parameter is highly affected by the input variables. The positive values of $r$ states the increasing effect of input parameters on the final objective and negative values of $r$ represents a decreasing trend for the dependency of the target to the inputs. Relevancy factor is obtained as follows:

$r=\frac{\sum_{i=1}^{N}\left(X_{k, i}-\bar{X}_{k}\right)\left(y_{i}-\bar{y}\right)}{\sqrt{\sum_{i=1}^{N}\left(X_{k, i}-\bar{X}_{k}\right)^{2} \sum_{i=1}^{N}\left(y_{i}-\bar{y}\right)^{2}}}$

$X_{k, i}$ expresses the $\mathrm{i}^{\text {th }}$ input, $\bar{X}_{k}$ denotes the mean value of the $\mathrm{k}^{\text {th }}$ input, $y_{i}$ indicates the $\mathrm{i}^{\text {th }}$ output, and $\bar{y}$ represents the mean value of output. $N$ is the overall population of data. The relevancy factors for all of the input data are illustrated in Fig. 19. The inlet temperature is monitored to be the most affecting variable in the efficiency of the PV/T system since the relevance factor of 0.36 was computed. 


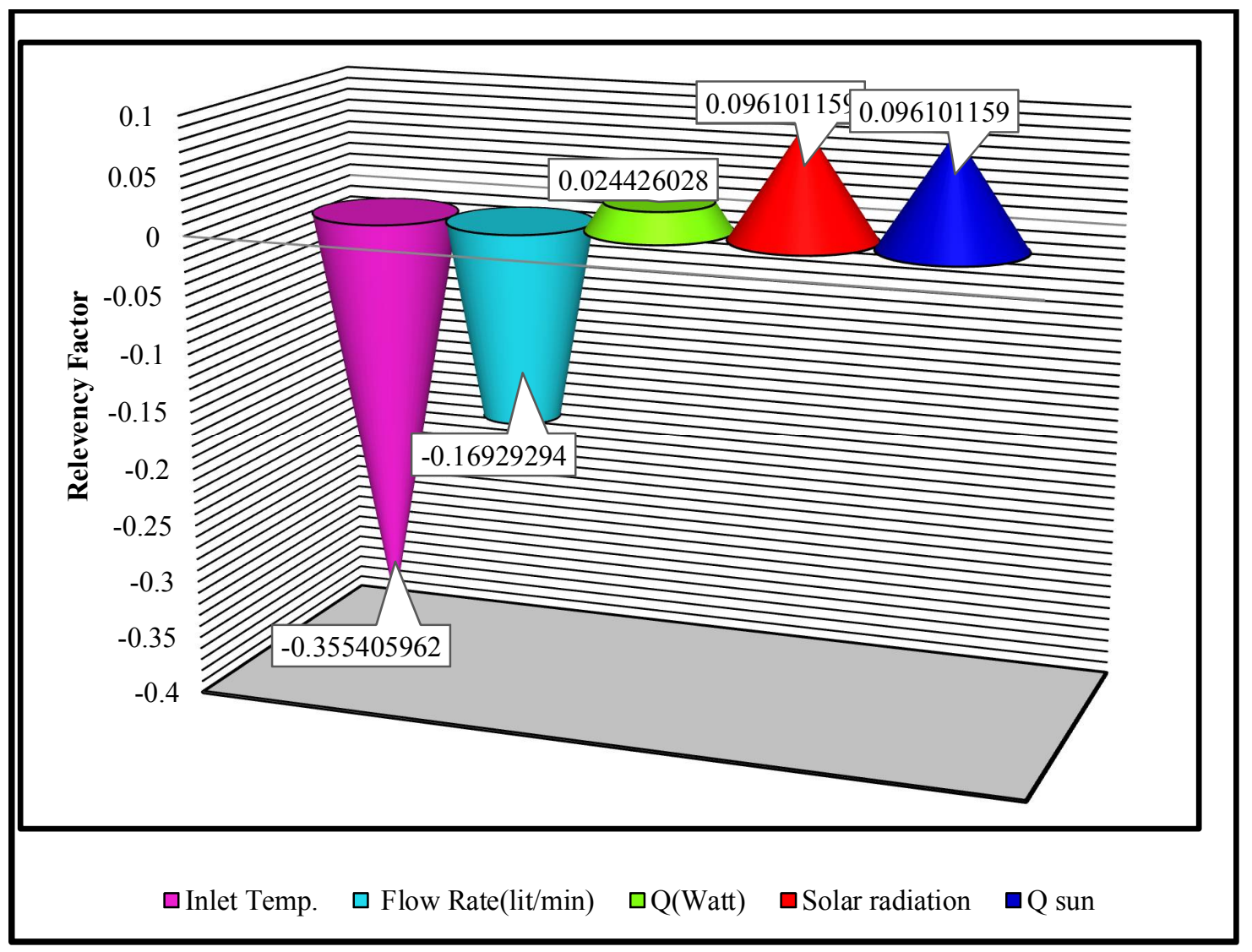

Figure 19: Relevancy factors for input varibles

\section{Conclusion}

Machine-based methods of MLP-ANN, RBF-ANN, ANFIS, and LSSVM were utilized to establish a mathematical model between efficiency of $\mathrm{PV} / \mathrm{T}$ collector and input parameters of inlet temperature, flow rate, heat, solar radiation, and heat of sun. The trustworthiness of the models in precise estimation of the efficiency was shown with Graphical and statistical approaches. In order to demonstrate the comprehensiveness of the models, the outlying recognition was performed. Further sensitivity analysis reports the effect of input data on the objective of the system's efficiency. Based on the sensitivity analysis, the water inlet temperature has the most effect on the efficiency of the PV/T system since it has the largest relevancy factor.

\section{References}


[1] Twidell J, Weir T. Renewable energy resources. Routledge; 2015.

[2] Kannan N, Vakeesan D. Solar energy for future world:-A review. Renew Sustain Energy Rev 2016;62:1092-105.

[3] Bong CPC, Ho WS, Hashim H, Lim JS, Ho CS, Tan WSP, et al. Review on the renewable energy and solid waste management policies towards biogas development in Malaysia. Renew Sustain Energy Rev 2017;70:988-98.

[4] Al-Maamary HMS, Kazem HA, Chaichan MT. Renewable energy and GCC States energy challenges in the 21st century: A review. Int J Comput Appl Sci IJOCAAS 2017;2:11-8.

[5] Lewis NS. Research opportunities to advance solar energy utilization. Science (80- ) 2016;351:aad1920.

[6] Modi A, Bühler F, Andreasen JG, Haglind F. A review of solar energy based heat and power generation systems. Renew Sustain Energy Rev 2017;67:1047-64.

[7] Sijm JPM. Cost and revenue related impacts of integrating electricity from variable renewable energy into the power system-A review of recent literature. Policy Stud 2017;2016:2015.

[8] Wagh S, Walke P V. REVIEW ON WIND-SOLAR HYBRID POWER SYSTEM. Int J Res Sci Eng 2017;3.

[9] Ahmad SHA, Saidur R, Mahbubul IM, Al-Sulaiman FA. Optical properties of various nanofluids used in solar collector: A review. Renew Sustain Energy Rev 2017;73:1014-30.

[10] Kumar A, Prakash O, Kaviti AK. A comprehensive review of Scheffler solar collector. Renew Sustain Energy Rev 2017;77:890-8. 
[11] Fuqiang W, Ziming C, Jianyu T, Yuan Y, Yong S, Linhua L. Progress in concentrated solar power technology with parabolic trough collector system: A comprehensive review. Renew Sustain Energy Rev 2017;79:1314-28.

[12] Pandey KM, Chaurasiya R. A review on analysis and development of solar flat plate collector. Renew Sustain Energy Rev 2017;67:641-50.

[13] Hussain F, Othman MYH, Sopian K, Yatim B, Ruslan H, Othman H. Design development and performance evaluation of photovoltaic/thermal (PV/T) air base solar collector. Renew Sustain Energy Rev 2013;25:431-41.

[14] Ali, Mumtaz, Asif Khan, and Naveed Ur Rehman. "Hybrid multiscale wind speed forecasting based on variational mode decomposition." International Transactions on Electrical Energy Systems 28, no. 1 (2018): e2466.

[15] Golestani, Samaneh, and Haidar Samet. "Polynomial - dynamic electric arc furnace model combined with ANN." International Transactions on Electrical Energy Systems (2018): e2561.

[16] Ranković, Aleksandar, Branko M. Maksimović, Andrija T. Sarić, and Uroš Lukič. "ANN based correlation of measurements in micro - grid state estimation." International Transactions on Electrical Energy Systems 25, no. 10 (2015): 2181-2202.

[17] Marquez R, Coimbra CFM. Forecasting of global and direct solar irradiance using stochastic learning methods, ground experiments and the NWS database. Sol Energy 2011;85:746-56.

[18] Moreno A, Gilabert MA, Martínez B. Mapping daily global solar irradiation over Spain: a 
comparative study of selected approaches. Sol Energy 2011;85:2072-84.

[19] Pandey AK, Tyagi V V, Jeyraj A, Selvaraj L, Rahim NA, Tyagi SK. Recent advances in solar photovoltaic systems for emerging trends and advanced applications. Renew Sustain Energy Rev 2016;53:859-84.

[20] Kramer K, Helmers H. The interaction of standards and innovation: Hybrid photovoltaicthermal collectors. Sol Energy 2013;98:434-9.

[21] Caner M, Gedik E, Keçebaş A. Investigation on thermal performance calculation of two type solar air collectors using artificial neural network. Expert Syst Appl 2011;38:1668-74.

[22] Varol Y, Koca A, Oztop HF, Avci E. Forecasting of thermal energy storage performance of Phase Change Material in a solar collector using soft computing techniques. Expert Syst Appl 2010;37:2724-32.

[23] Baghban A, Ahmadi MA, Shahraki BH. Prediction carbon dioxide solubility in presence of various ionic liquids using computational intelligence approaches. J Supercrit Fluids $2015 ; 98: 50-64$.

[24] Ghazani SHHN, Baghban A, Mohammadi AH, Habibzadeh S. Absorption of $\mathrm{CO}<\inf >2</$ inf $>$-rich gaseous mixtures in ionic liquids: A computational study. J Supercrit Fluids 2018;133. doi:10.1016/j.supflu.2017.10.024.

[25] Baghban A, Mohammadi AH, Taleghani MS. Rigorous modeling of CO 2 equilibrium absorption in ionic liquids. Int J Greenh Gas Control 2017;58:19-41.

[26] Brown M, Harris CJ. Neurofuzzy adaptive modelling and control. Prentice Hall; 1994.

[27] Lin C-T, Lee CSG. Neural fuzzy systems. PTR Prentice Hall 1996. 
[28] Schalkoff RJ. Artificial neural networks. vol. 1. McGraw-Hill New York; 1997.

[29] Yegnanarayana B. Artificial neural networks. PHI Learning Pvt. Ltd.; 2009.

[30] Mitchell TM. Artificial neural networks. Mach Learn 1997;45:81-127.

[31] Haykin S. Neural networks: a comprehensive foundation. Prentice Hall PTR; 1994.

[32] Haykin SS, Haykin SS, Haykin SS, Haykin SS. Neural networks and learning machines. vol. 3. Pearson Upper Saddle River, NJ, USA:; 2009.

[33] Wasserman PD. Advanced methods in neural computing. John Wiley \& Sons, Inc.; 1993.

[34] Park J, Sandberg IW. Approximation and radial-basis-function networks. Neural Comput $1993 ; 5: 305-16$.

[35] Suykens JAK, Van Gestel T, De Brabanter J, De Moor B, Vandewalle J. Least squares support vector machines, World Scientific Publishing, Singapore 2002.

[36] Pelckmans K, Suykens JAK, Van Gestel T, De Brabanter J, Lukas L, Hamers B, et al. LSSVMlab: a matlab/c toolbox for least squares support vector machines. Tutorial KULeuvenESAT Leuven, Belgium 2002;142:1-2.

[37] Suykens JAK, Van Gestel T, De Brabanter J. Least squares support vector machines. World Scientific; 2002.

[38] Ye J, Xiong T. Svm versus least squares svm. Int. Conf. Artif. Intell. Stat., 2007, p. 64451.

[39] Vapnik V, Golowich SE, Smola AJ. Support vector method for function approximation, regression estimation and signal processing. Adv. Neural Inf. Process. Syst., 1997, p. 281- 
7.

[40] Andrews DF. Plots of High-Dimensional Data. Biometrics 1972;28:125-36. doi: $10.2307 / 2528964$.

[41] Rousseeuw PJ, Leroy AM. Robust regression and outlier detection. vol. 589. John wiley \& sons; 2005. 